\title{
QUASISYMMETRIES OF THE BASILICA AND THE THOMPSON GROUP
}

\author{
MIKHAIL LYUBICH AND SERGEI MERENKOV
}

\begin{abstract}
We give a description of the group of all quasisymmetric self-maps of the Julia set of $f(z)=z^{2}-1$ that have orientation preserving homeomorphic extensions to the whole plane. More precisely, we prove that this group is the uniform closure of the group generated by the Thompson group of the unit circle and an inversion. Moreover, this result is quantitative in the sense that distortions of the approximating maps are uniformly controlled by the distortion of the given map.
\end{abstract}

\section{INTRODUCTION}

Quasisymmetric geometry of fractal sets has attracted substantial interest in recent years. A natural invariant in this category is the group of quasisymmetries of the set. One can roughly classify such a set as "little quasisymmetric" or "highly quasisymmetric", depending on whether this group is finite or infinite. We are interested in this dichotomy for Julia sets of rational maps. In our previous paper [BLM], joint with Mario Bonk, we described a class of little quasisymmetric Julia sets that are Sierpiński carpets. The goal of this paper is to give an example of a highly quasisymmetric Julia set, the basilica (see Figure 1), and to describe its group of quasisymmetries.

To formulate the main result, let us give quick definitions of the main objects, referring to the main body of the paper for their precise versions.

Let $f: \mathbb{C} \rightarrow \mathbb{C}$ be a polynomial of degree $\geq 2$. Its filled Julia set $\mathcal{K}(f)$ is defined as the set of non-escaping points, and the Julia set $\mathcal{J}(f)$ is defined as the boundary of $\mathcal{K}(f)$.

The basilica is the filled Julia set of the quadratic polynomial $f: z \mapsto$ $z^{2}-1$ This polynomial has a superattracting cycle $\gamma=\{0,-1\}$ of period two, and int $\mathcal{K}(f)$ coincides with the basin of this cycle. By

M.L. was supported by NSF grants DMS-1007266, DMS-1301602, DMS-1600519. S.M. is grateful for the support by the Simons Foundation at the IMS.

${ }^{1}$ In this paper, we refer informally to the corresponding Julia set $\mathcal{J}$ also as "basilica". 


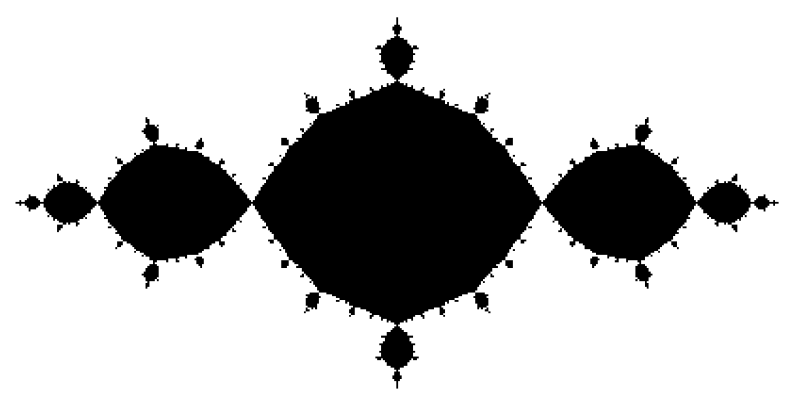

Figure 1. The filled Julia set of $f(z)=z^{2}-1$.

definition, the immediate basin of $\gamma$ is the union of two components $U_{0}$ and $U_{-1}$ of the basin containing 0 and -1 , respectively. These components are Jordan discs, and the Riemann mapping $\phi_{0}: U_{0} \rightarrow \mathbb{D}$, such that $\phi_{0}(0)=0, \phi_{0}^{\prime}(0)<0$, brings the return map $f^{2}: U_{0} \rightarrow U_{0}$ to the monomial form $g: z \mapsto z^{2}$.

Given a homeomorphism $\eta:[0, \infty) \rightarrow[0, \infty)$, a homeomorphism $h: \mathcal{J}(f) \rightarrow \mathcal{J}(f)$ is called a quasisymmetry with distortion function $\eta$ (or $\eta$-quasisymmetry) if

$$
\frac{|h(u)-h(v)|}{|h(u)-h(w)|} \leq \eta\left(\frac{|u-v|}{|u-w|}\right),
$$

for all triples of distinct points $u, v$, and $w$ in $\mathcal{J}(f)$. In fact, this definition is applicable to a homeomorphism $h: X \rightarrow X$ of any metric space $X$, with the Euclidean distance replaced by the corresponding metric. Moreover, in the case of the complex plane, $X=\mathbb{C}, \eta$-quasisymmetry is equivalent to $K$-quasiconformality, quantitatively (provided the map is normalized at two points). We say that a homeomorphism of $\mathcal{J}(f)$ is topologically extendable if it has an extension to an orientation preserving homeomorphism of $\mathbb{C}$.

The Thompson group $T$ is defined as the group of piecewise linear (in the angular coordinate) homeomorphisms of the unit circle $\mathbb{T}$ with breaks at some dyadic points and slopes equal to integer powers of 2 . By means of the Riemann mapping $\phi_{0}$, we can make this group act on $\partial U_{0}$. In this paper, we show that this action admits an extension to an action by quasisymmetries of the basilica Julia set $\mathcal{J} \equiv \mathcal{J}(f)$. We also construct one more quasisymmetry of $\mathcal{J}$, an involution $\iota$, which permutes the components $U_{0}$ and $U_{-1}$ of the immediate basin. Let $\hat{T}$ be the extended Thompson group of quasisymmetries of $\mathcal{J}$ generated by $T$ and $\iota$. Our main result asserts that this group generates quantitatively the whole group of topologically extendable quasisymmetries of $\mathcal{J}$ : 
Theorem 1.1. For any distortion function $\eta$ there exists a distortion function $\eta^{\prime}$, such that for any topologically extendable $\eta$-quasisymmetry $\xi$ of the basilica Julia set $\mathcal{J}$ there exists a sequence of $\eta^{\prime}$-quasisymmetries $\tau_{n}$ of $\mathcal{J}$ that belong to the extended Thompson group $\hat{T}$ and uniformly converge to $\xi$.

Thus, the basilica is highly quasisymmetric. As we have already mentioned, this contrasts with the result of [BLM] concerning Sierpiński Julia sets. Note that the standard Sierpiński carpet is little quasisymmetric as well [BM]. On the other hand, it was shown in [Me that the "slit carpet" is highly quasisymmetric, and the corresponding group of quasisymmetries bears some similarity with the Thompson group.

Note that the limit sets of non-elementary Kleinian groups are highly symmetric, as they admit infinite groups of Möbius symmetries. Let us say that a compact subset $K \subseteq \widehat{\mathbb{C}}$ of the sphere is rigid if all its quasisymmetries are Möbius. It was shown in [BKM that "Schottky sets" of zero area are rigid, contrasting again with our result.

Let us also mention a recent work of J. Belk and B. Forrest $[\mathrm{BF}]$ who studied a Thompson-like group of circle homeomorphisms that preserve the invariant lamination of the basilica, and hence descend to homeomorphisms of the basilica itself. Our result implies that these homeomorphisms are quasisymmetric (and, in fact, form a dense subgroup of basilica quasisymmetries). Unlike $[\mathrm{BF}$, we approach the problem from "inside" of the basilica, beginning with the Thompson group acting on the immediate basin.

Let us also note that a minor modification of our construction (that does not need Lemma 7.1) would produce the following result. If $\xi$ in Theorem 1.1 is only assumed to be a homeomorphic self-map of $\mathcal{J}$ that has an orientation preserving homeomorphic extension to $\mathbb{C}$, then there is a sequence of maps $\tau_{n}, n=1,2, \ldots$, in $\hat{T}$ that converge uniformly on $\mathcal{J}$ to $\xi$. So we can describe the full group of extendable homeomorphisms of $\mathcal{J}$ as the uniform closure of $\hat{T}$. However, even though each map $\tau_{n}$ is quasisymmetric, one generally does not have a uniform control on their distortion functions. The key technical step of our construction is Lemma 7.1 that provides us with uniform quasisymmetric control of approximating maps.

Let us finally note that our method extends in a straightforward way to hyperbolic Julia sets in the "main molecule" of the Mandelbrot set (obtained from $z^{2}$ through a finite cascade of satellite bifurcations), e.g., to the Douady rabbit. 
1.1. Notation and terminology. Throughout the paper we denote $\mathbb{N} \cup\{0\}$ by $\mathbb{N}_{0}$. We let $\mathbb{D}$ be the open unit disc in the complex plane $\mathbb{C}$, and let $\mathbb{T}=\partial \mathbb{D}$ be the unit circle in $\mathbb{C}$.

In what follows, we label a point $z=e(\theta)=e^{2 \pi i \theta} \in \mathbb{T}$ by $\theta \in \mathbb{R} / \mathbb{Z}$. In other words, the angular measure on the circle $\mathbb{T}$ is scaled so that its total length is equal to 1 , which is standard in dynamics. Points $e\left((2 k+1) / 2^{n}\right) \in \mathbb{T}, k=0,1, \ldots, 2^{n-1}-1, n \in \mathbb{N}$, are called dyadic points of level $n$, and $e(0)$ is the dyadic point of level 0 . Taking the union of dyadic points of levels $m \leq n$, we obtain $2^{n}$ points $e\left(l / 2^{n}\right)$, $l=0,1, \ldots, 2^{n}-1$, that tessellate the circle into $2^{n}$ (closed) intervals $I_{l}^{n}$. We will refer to these intervals as dyadic intervals of level $n$.

Let $g$ denote the map $z \mapsto z^{2}$, which is doubling in the angular coordinate of $\mathbb{T}$, i.e., $\theta \mapsto 2 \theta \quad \bmod \mathbb{Z}$. The dyadic points $z \in \mathbb{T}$ are dynamically identified as the iterated preimages of the fixed point 1 , with the level equal to the smallest $n \in \mathbb{N}_{0}$ such that $g^{n}(z)=1$.

\section{Dyadic Subdivision of Bounded Fatou COMponents}

The reader can consult [CG, Mi] for a general introduction to the iteration theory of rational functions, and [DH, L] for particular features of the dynamics of quadratic polynomials.

The Fatou set is the complement of the Julia set, $\mathcal{F}(f)=\mathbb{C} \backslash \mathcal{J}(f)$. Its connected components are called Fatou components. Bounded Fatou components can also be identified as the connected components of the interior of the filled Julia set $\mathcal{K}(f)$.

As we have already mentioned, the basilica map $f(z)=z^{2}-1$ is specified by the property that it has a superattracting periodic cycle $\{0,-1\}$ of period two. In particular, it is a postcritically finite hyperbolic map. We let $U_{0}$ and $U_{-1}$ be the Fatou components of $f$ that contain 0 and -1 , respectively. Both of these components are Jordan discs. The map $f$ takes $U_{0}$ onto $U_{-1}$ as a double branched covering, and it takes $U_{-1}$ back onto $U_{0}$ conformally.

Let $\phi_{0}:\left(\overline{U_{0}}, 0, \alpha\right) \rightarrow(\overline{\mathbb{D}}, 0,1)$ be the Böttcher coordinate of $U_{0}$ (which coincides with the appropriately normalized Riemann uniformization of $U_{0}$ ), homeomorphically extended to the boundary. It conjugates the return map $f^{2}: \overline{U_{0}} \rightarrow \overline{U_{0}}$ to the monomial map $g: \overline{\mathbb{D}} \rightarrow \overline{\mathbb{D}}, z \mapsto z^{2}$.

The left-most point of the closure $\overline{U_{0}}$ on the real line is a fixed point $\alpha$ of $f$, is equal to $(1-\sqrt{5}) / 2$. It is also the unique point of intersection of $\overline{U_{0}}$ and $\overline{U_{-1}}$, called the root of each of these components. Moreover, $\alpha$ is a global cut-point for the Julia set $\mathcal{J}$ : puncturing $\alpha$ out results in breaking $\mathcal{J}$ into two connected components. All the preimages of $\alpha$ under the iterates of $f$ are therefore also global cut-points. The other 
fixed point of $f$ is $\beta=(1+\sqrt{5}) / 2$. It is the right-most point of the intersection of $\mathcal{J}$ with the real line.

Every bounded Fatou component $U$ of $f$ eventually, i.e., under a certain iterate of $f$, lands in the cycle $\left\{U_{0}, U_{-1}\right\}$. In fact, for each $U$ there exists a unique $n \in \mathbb{N}_{0}$ such that $f^{n}: U \rightarrow U_{0}$ is a conformal map. We call such $n$ the dynamical distance from $U$ to $U_{0}$. It follows that all bounded Fatou components of $f$ are Jordan discs as well. Then the map $\phi_{U}=\phi_{0} \circ f^{n}$ is a conformal map of $U$ onto $\mathbb{D}$ that extends to a homeomorphism $\bar{U} \rightarrow \overline{\mathbb{D}}$. It is called the Böttcher coordinate of $U$. The root $\alpha_{U} \in \partial U$ of $U$ is defined as the preimage of the root $\alpha \in U_{0}$ under $f^{n}$, or equivalently, as the point in $\partial U$ whose Böttcher coordinate $\phi_{U}\left(\alpha_{U}\right)$ is equal to $1 \in \mathbb{T}$.

Similarly, we define dyadic points on $\partial U$ as points whose Böttcher coordinates are dyadic. Dyadic points of level $\leq m$ induce a level $m$ dyadic subdivision of $\partial U$. It can also be described as follows: for a bounded Fatou component $U$ of dynamical distance $n \geq 0$ from $U_{0}$ and for an integer number $m \geq 0$, the full preimage $f^{-(n+2 m)}(\alpha)$ gives the dyadic decomposition of $\partial U$ of level $m$ that consists of $2^{m}$ points.

The basilica filled Julia set $\mathcal{K} \equiv \mathcal{K}(f)$ has the following combinatorial structure of a (non-locally finite) tree $\mathfrak{T}$. The vertices of $\mathfrak{T}$ are the bounded Fatou components of $f$. (In what follows we make no distinction between bounded Fatou components and the vertices of $\mathfrak{T}$.) Two vertices $U$ and $V$ are connected by an edge $E$ if and only if they touch, i.e., their closures intersect. Let $\delta(U)$ stand for the combinatorial distance in this tree from a vertex $U$ to the main vertex $U_{0}$. If two vertices $U$ and $V$ are adjacent, then $|\delta(U)-\delta(V)|=1$. Under these circumstances, if $\delta(U)=\delta(V)+1$ then $V$ is called the principal vertex of $E$, while $U$ is called a satellite of $V$.

The main edge of $\mathfrak{T}$, denoted $E_{0}$, is the edge that connects $U_{0}$ to $U_{-1}$ via their common root $\alpha$. The higher level edges are described as follows:

Lemma 2.1. Let $U$ and $V$ be distinct bounded Fatou components that touch at a point $z$. Let $n \in \mathbb{N}_{0}$ and $m \in \mathbb{N}_{0}$ be the dynamical distances from $U$ and $V$ to $U_{0}$, respectively, with $n \geq m$. Then $U$ is a satellite of $V$ and the root of $U$ is $z$.

Proof. The distances $m$ and $n$ cannot be equal because otherwise we get a contradiction with the fact that $f^{m}$ is conformal in a neighborhood of $z$. Application of $f^{k}, k \leq m$, preserves the differences in dynamical and in combinatorial distances from $U$ and $V$ to $U_{0}$, respectively. This implies that the combinatorial distance from $V$ to $U_{0}$ in $\mathfrak{T}$ is one less than that from $U$ to $U_{0}$. Now, $f^{m}(U)$ is a bounded Fatou 
component that touches $f^{m}(V)=U_{0}$. The point of the intersection is $f^{m}(z)$, and $n-m \in \mathbb{N}$ is the dynamical distance from $f^{m}(U)$ to $U_{0}$. Thus $f^{n-m-1}\left(f^{m}(U)\right)$ is either $U_{-1}$ or $U_{1}$, the bounded Fatou components that contain -1 and 1 , respectively. In either case we must have $f^{n-m-1}\left(U_{0}\right)=U_{0}$ because $f$ is at most two to one in each Fatou component. Hence $f^{n-1}(z)=f^{n-m-1}\left(f^{m}(z)\right)$ is either $\alpha$ or $-\alpha$, and thus $f^{n}(z)=\alpha$, i.e., $z$ is the root of $U$.

Thus, every edge $E$ can be labeled by the principal component $V$ and a dyadic number $d \in \mathbb{T}$ representing the point on $\partial V$ where the satellite component $U$ touches $V$.

\section{Dynamical partitions of the Julia set $\mathcal{J}$}

Let $U_{\infty}$ denote the basin at infinity, i.e., the unbounded Fatou component of $f$, and let $\phi_{\infty}$ be the Böttcher coordinate of $U_{\infty}$. Namely, $\phi_{\infty}$ is the conformal map of $U_{\infty}$ onto the complement of the closed unit disc $\mathbb{C} \backslash \overline{\mathbb{D}}$ conjugating $f$ to $g: z \mapsto z^{2}$.

The external ray $\rho^{\theta}$ with angle $\theta \in \mathbb{R} / \mathbb{Z}$ is defined as the pullback of the straight ray $\rho^{\theta}=\{r e(\theta): 1<r<\infty\}$ under the Böttcher map $\phi_{\infty}$. The external rays form an invariant foliation with $f$ acting by the angle-doubling: $f\left(\rho^{\theta}\right)=\rho^{2 \theta}$.

It is known that the Julia set of a hyperbolic map $f$ is locally connected, so the inverse Böttcher map $\psi_{\infty}=\phi_{\infty}^{-1}: \mathbb{D} \rightarrow U_{\infty}$ extends continuously to the boundary, and so induces a continuous boundary map of $\mathbb{T}$ onto $\mathcal{J}$. It follows that any ray $\rho^{\theta}$ lands at some point $z_{\theta} \in \mathcal{J}$. For a circle arc $I \subseteq \mathbb{T}$, we call

$$
\psi_{\infty}(I)=\left\{z_{\theta}: e(\theta) \in I\right\}
$$

a Julia arc.

In the case of the basilica, there exist exactly two rays, $\rho^{ \pm 1 / 3}$, that land at the fixed point $\alpha$. They bound two (open) sectors, $S_{0} \supset U_{0}$ and $S_{\alpha} \supset U_{-1}$. The latter sector is also called the wake rooted at $\alpha$. The intersection $\mathcal{K}_{\alpha}:=\left(\mathcal{K} \cap S_{\alpha}\right) \cup\{\alpha\}$ (with the added root) is called the limb of $\mathcal{K}$ rooted at $\alpha$. We denote by $\mathcal{J}_{\alpha}$ the Julia arc $\partial \mathcal{K}_{\alpha}$.

This picture can be spread around to the iterated preimages of $\alpha$. Let $z \in \mathcal{J}$ and $f^{n}(z)=\alpha$ for some $n \in \mathbb{N}_{0}$. We choose the smallest moment $n$ like this. Then $z$ is the landing point of exactly two rays, $f^{n}$-preimages of $\rho^{ \pm 1 / 3}$. They bound the unique (open) sector $S_{z}$ that does not contain $U_{0}$; it is called the wake rooted at $z$. Moreover, if $n \geq 1$, then $f^{n}$ conformally maps $S_{z}$ onto $S_{0}$. Let $\mathcal{K}_{z}:=\left(S_{z} \cap \mathcal{K}\right) \cup\{z\}$ be the limb of $\mathcal{K}$ rooted at $z$, and let $\mathcal{J}_{z}:=\partial \mathcal{K}_{z}$ be the corresponding Julia arc. Below we may also refer to $\mathcal{J}_{z}$ as a limb rooted at $z$. 
Let $\mathcal{R}_{n}, n \in \mathbb{N}_{0}$, be the family of all external rays in $U_{\infty}$ that land at points of the full preimage $f^{-n}(\alpha)$. Note that each external ray of $\mathcal{R}_{n}$ lands on the boundaries of exactly two bounded Fatou components, adjacent components of the tree $\mathfrak{T}$.

We say that distinct external rays $\rho_{1}$ and $\rho_{2}$ of $\mathcal{R}_{n}$ are adjacent if they are not separated in $U_{\infty}$ by other external rays of $\mathcal{R}_{n}$ (in other words, the angles $\theta_{1}, \theta_{2}$ are adjacent points of the set $g^{-n}\{ \pm 1 / 3\} \subset \mathbb{T}$ ).

Lemma 3.1. If $\rho_{1}, \rho_{2} \in \mathcal{R}_{n}$ are adjacent external rays, then there exists a bounded Fatou component $U$ of $f$ such that $\rho_{1}$ and $\rho_{2}$ land at boundary points of $U$.

Proof. We apply induction. The statement is trivially true for $n=0$. Assuming that the statement is true for $n-1$, where $n \geq 1$, let $\rho_{1}$ and $\rho_{2}$ be two adjacent external rays of $\mathcal{R}_{n}$. If we apply $f$ to $\rho_{1}$ and $\rho_{2}$, we obtain two external rays of $\mathcal{R}_{n-1}$ that are necessarily adjacent. Otherwise, we would apply $f^{-1}$ to the region in $U_{\infty}$ between $f\left(\rho_{1}\right)$ and $f\left(\rho_{2}\right)$ that contains a separating external ray to get a contradiction. Note that in the Böttcher coordinate of $U_{\infty}$ the arc on $\mathbb{T}$ that corresponds to the landing points of $\rho_{1}$ and $\rho_{2}$ and does not contain the landing points of other rays in $\mathcal{R}_{n}$ has angular length at most $1 / 3<1 / 2$, and hence $f$ is conformal in the region of $U_{\infty}$ between $\rho_{1}$ and $\rho_{2}$ that does not contain other external rays of $\mathcal{R}_{n}$. Therefore, $f\left(\rho_{1}\right)$ and $f\left(\rho_{2}\right)$ land on the boundary of the same bounded Fatou component $U$. If $U=U_{-1}$, then $\rho_{1}$ and $\rho_{2}$ must land on the boundary of $U_{0}$, because $U_{0}$ is the only preimage of $U_{-1}$ under $f$. If $U \neq U_{-1}$, any branch of the map $f^{-1}$ restricted to $U$ is conformal onto another bounded Fatou component $V$, and there are exactly two such branches because $f$ has degree 2 . Suppose $\rho_{1}$ lands on the boundary of $V$. The branch $f^{-1}$ that takes $U$ onto $V$ extends conformally across the arc $\omega$ on the boundary of $U$ between the landing points of $f\left(\rho_{1}\right)$ and $f\left(\rho_{2}\right)$ into the region $R$ in $\mathbb{C} \backslash \bar{U}$ that satisfies the following properties. The arc $\omega$ does not contain other landing points of external rays in $\mathcal{R}_{n-1}$, and the region $R$ is bounded by $\omega, f\left(\rho_{1}\right)$, and $f\left(\rho_{2}\right)$. The image of $f\left(\rho_{2}\right)$ under this branch of $f^{-1}$ is then necessarily the external ray $\rho_{2}$, and we are done.

For $n \in \mathbb{N}_{0}$, let:

- $D_{n}$ be the $n$-fold preimage $g^{-n}\{ \pm 1 / 3\} \subset \mathbb{T}$ (consisting of $2^{n+1}$ points); $-P_{n}$ be the tiling of the circle $\mathbb{T}$ by the points of $D_{n}$ (comprising $2^{n+1}$ closed arcs $\left.I_{k} \subset \mathbb{T}\right)$;

- $\Pi_{n}=\psi_{\infty}\left(P_{n}\right)$ be the corresponding tiling of $\mathcal{J}$ (comprising $2^{n+1}$ closed Julia arcs $J_{k}=\psi_{\infty}\left(I_{k}\right) \subset \mathcal{J}$ with endpoints at preimages of the fixed point $\alpha$ of level $\leq n$ ). 
Each set $J_{k}$ corresponds to a pair of adjacent external rays of $\mathcal{R}_{n}$, as follows. Let $\rho_{1}$ and $\rho_{2}$ be two adjacent external rays of $\mathcal{R}_{n}$ whose landing points are $z_{1}$ and $z_{2}$, respectively. There are two cases that need to be considered: either $z_{1}=z_{2}$ or $z_{1} \neq z_{2}$.

Case 1. If $z=z_{1}=z_{2}$, this point is the root of a unique bounded Fatou component $U$ unless $z=\alpha$, the fixed point of $f$. This follows from Lemma 2.1. If $z=\alpha$, we choose $U=U_{-1}$. The Julia arc $J_{k}$ of $\Pi_{n}$ that corresponds to $\rho_{1}, \rho_{2}$ is $\mathcal{J}_{z}$.

Case 2. Now assume that $z_{1} \neq z_{2}$ and let $U$ be a bounded Fatou component of $f$ whose boundary contains $z_{1}$ and $z_{2}$. Such $U$ exists by Lemma 3.1. Let $\omega$ be the arc on $\partial U$ between $z_{1}$ and $z_{2}$ that contains no other landing points of external rays in $\mathcal{R}_{n}$. Let $R$ be the region in $\mathbb{C} \backslash \bar{U}$ that is bounded by $\omega, \rho_{1}$, and $\rho_{2}$. The Julia arc $J_{k}$ that corresponds to the pair $\rho_{1}, \rho_{2}$ is the closure of the intersection of $\mathcal{J}$ with the region $R$.

It follows immediately from the definition that the map $f^{n}$ takes each Julia arc $J_{k}$ of $\Pi_{n}$ onto the closure of one of the two connected components of $\mathcal{J} \backslash\{\alpha\}$ : either $\mathcal{J}_{0}$ that contains the boundary of $U_{0}$ or $\mathcal{J}_{\alpha}$ that contains the boundary of $U_{-1}$. The first case occurs when the landing points $z_{1}$ and $z_{2}$ are the same. In this case the map $f^{n}$ is one to one. In the other case, i.e., $z_{1} \neq z_{2}$, the preimage of each point $z$ of $\mathcal{J}_{\alpha}$ under $f^{n}$ is a singleton except if $z=\alpha$, when the preimage consists of two points.

Lemma 3.2. The set $D_{n}, n \geq 0$, is the set of $2^{n+1}$ points on the unit circle such that the angular lengths of complimentary intervals alternate between $1 /\left(3 \cdot 2^{n}\right)$ and $2 /\left(3 \cdot 2^{n}\right)$. In particular, there exists a constant $L \geq 1$ independent of $n$, and an orientation preserving piecewise linear L-bi-Lipschitz map $\psi$ of $\mathbb{T}$ whose break points are points of $D_{n}$, and such that $\psi\left(D_{n}\right)$ is a set of $2^{n+1}$ points on the unit circle such that all complementary intervals have equal lengths.

Proof. Let $I$ and $J$ be two adjacent complementary intervals at level $n$. Application of the map $g(z)=z^{2}$ doubles the lengths of each one of them and keeps them adjacent. Because for $n=0$ the points $1 / 3$ and $2 / 3$ satisfy the desired property and form a 2-cycle under the dynamics of $g$, the first part of the claim follows from induction. The second part follows from the observation that in order to make all complementary intervals to have the same length, one needs to scale the complementary intervals of length $1 /\left(3 \cdot 2^{n}\right)$ by $3 / 2$ and the complementary intervals of length $2 /\left(3 \cdot 2^{n}\right)$ by $3 / 4$. In particular, $L=3 / 2$.

Let us finish this section with a description of a pinched disk topological model for the basilica. Let $d$ be the diameter of $\mathbb{D}$ with one 
endpoint at $e(1 / 3)$, and let $\mathbb{D}_{ \pm}$be the corresponding semi-disks. For the sake of definiteness we assume that $e(2 / 3)$ is contained in $\mathbb{D}_{-}$. Let us connect the points $e(1 / 3)$ and $e(2 / 3)$ of $D_{0}$ with the hyperbolic geodesic $\gamma^{0} \subset \overline{\mathbb{D}}$. The points of $D_{1}$ split into two symmetric pairs, one contained in $\overline{\mathbb{D}_{+}}$, the other contained in $\overline{\mathbb{D}_{-}}$. Each of these pairs can be connected with a hyperbolic geodesic. Since $\{e(1 / 3), e(2 / 3)\}$ is a periodic cycle for the map $g$, one of these geodesics, namely the one contained in $\overline{\mathbb{D}_{-}}$, is the geodesic $\gamma^{0}$. We denote the other geodesic by $\gamma^{1}$. The full preimage of $\partial \gamma^{1}$ under the doubling map $g$ consists of two pairs of points in $D_{2}$, one contained in $\mathbb{D}_{+}$, the other contained in $\mathbb{D}_{-}$. Connecting each of these pairs with a hyperbolic geodesic, we obtain two new geodesics in $D_{2} \backslash D_{1}$, denoted by $\gamma_{+}^{2}$ and $\gamma_{-}^{2}$. By the same procedure, for each geodesic $\gamma_{\epsilon}^{2}, \epsilon= \pm$, we obtain two new geodesics ("pullbacks" of $\gamma_{\epsilon}^{2}$ ), one in $\mathbb{D}_{+}$and the other contained in $\mathbb{D}_{-}$, which we denote by $\gamma_{\left(\epsilon_{1} \epsilon_{2}\right)}^{3}, \epsilon_{i} \in \pm, i=1,2$. Proceeding this way, for each level $n \in \mathbb{N}$, we can construct $2^{n-1}$ hyperbolic geodesics $\gamma_{\bar{\epsilon}}^{n}, \bar{\epsilon}=\left(\epsilon_{1} \ldots \epsilon_{n-1}\right)$, $\epsilon_{i} \in \pm$, paring the points of $D_{n} \backslash D_{n-1}$. It is easy to show that all geodesics $\gamma_{\bar{\epsilon}}^{n}$ are disjoint. One can also show that together they form a closed subset $Q$ of $\overline{\mathbb{D}}$. This subset (endowed with a partition into the geodesics $\gamma_{\bar{\epsilon}}^{n}$ ) is called the basilica lamination; see Figure2.

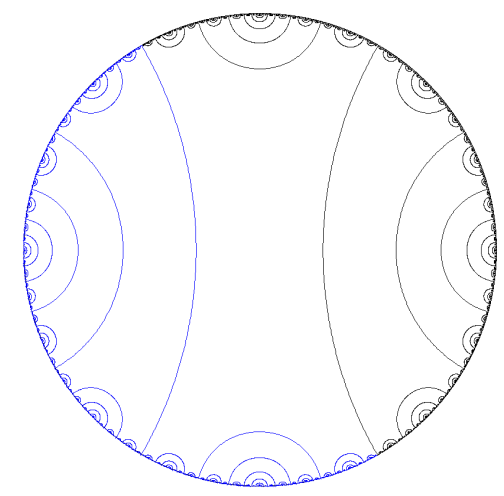

Figure 2. The basilica lamination ${ }^{2}$

Let us consider an equivalence relation $\underset{f}{\sim}$ on $\mathbb{C}$ whose classes are either the geodesics of the basilica lamination or single points.

\footnotetext{
${ }^{2}$ Source: https://commons.wikimedia.org/wiki/File:Basilica_lamination.png
} 
Theorem 3.3. (see, e.g., [L, Theorem 24.33]) The quotient of $(\mathbb{C}, \overline{\mathbb{D}})$ by the equivalence relation $\underset{f}{\sim}$ is homeomorphic to $(\mathbb{C}, \mathcal{K})$. Moreover, this homeomorphism coincides with the inverse Bötcher coordinate $\psi_{\infty}$ on $\mathbb{C} \backslash \overline{\mathbb{D}}$ and sends the geodesics $\gamma_{\bar{\epsilon}}^{n}$ to the corresponding level $n$ preimages of the $\alpha$-fixed point.

The equivalence relation $\underset{f}{\sim}$ induces an equivalence relation on the circle $\mathbb{T}$ that pairs points of $D_{\infty}:=\bigcup D_{n}$, where $\theta_{1} \underset{f}{\sim} \theta_{2}$ if and only if the rays $\rho^{\theta_{1}}$ and $\rho^{\theta_{2}}$ land at the same point of $\mathcal{J}$. We will refer to it as the basilica lamination of $\mathbb{T}$. (This "lamination" has zerodimensional leaves.) Obviously, the basilica lamination of $\mathbb{T}$ contains the same amount of information as the basilica geodesic lamination of $\overline{\mathbb{D}}$.

For the sake of reference, let us state a simple lemma:

Lemma 3.4. Any homeomorphism $\xi: U_{\infty} \cup \mathcal{J} \rightarrow U_{\infty} \cup \mathcal{J}$ lifts (via the extended inverse Böttcher coordinate $\left.\psi_{\infty}: \mathbb{C} \backslash \mathbb{D} \rightarrow U_{\infty} \cup \mathcal{J}\right)$ to a homeomorphism $h: \mathbb{C} \backslash \mathbb{D} \rightarrow \mathbb{C} \backslash \mathbb{D}$ that preserves the basilica lamination of $\mathbb{T}$, and whose restriction $\xi_{\infty}$ to $\mathbb{T}$ satisfies

$$
\psi_{\infty} \circ \xi_{\infty}=\xi \circ \psi_{\infty}
$$

Conversely, any homeomorphism $h: \mathbb{C} \backslash \mathbb{D} \rightarrow \mathbb{C} \backslash \mathbb{D}$ that preserves the basilica lamination of $\mathbb{T}$ descends to a homeomorphism $\xi: U_{\infty} \cup \mathcal{J} \rightarrow$ $U_{\infty} \cup \mathcal{J}$.

Proof. On $\mathbb{C} \backslash \overline{\mathbb{D}}$, the map $h$ is defined as $\phi_{\infty} \circ \xi \circ \psi_{\infty}$. By the Carathéodory Theory, the closure $\mathbb{C} \backslash \mathbb{D}$ is naturally homeomorphic to the prime end compactification $\mathrm{cl}^{C} U_{\infty}$ of $U_{\infty}$. The definition of prime ends easily implies that any homeomorphism $\xi: U_{\infty} \cup \mathcal{J} \rightarrow U_{\infty} \cup \mathcal{J}$ induces a homeomorphism of $\mathrm{cl}^{C} U_{\infty}$. It follows that $h$ extends continuously to the unit circle $\mathbb{T}$. This extension respects the basilica lamination of $\mathbb{T}$ since $\xi$ respects the landing pairing between the rays. Equation 3.1 follows by equating the boundary values of $\psi_{\infty} \circ h$ and $\xi \circ \psi_{\infty}$.

Conversely, any homeomorphism $h: \mathbb{C} \backslash \mathbb{D} \rightarrow \mathbb{C} \backslash \mathbb{D}$ that preserves the basilica lamination on $\mathbb{T}$ descends to a homeomorphism of the quotient $(\mathbb{C} \backslash \mathbb{D}) / \underset{f}{\sim}$. By Theorem 3.3, the latter is naturally homeomorphic to $U_{\infty} \cup \mathcal{J}$, providing a desired homeomorphism $\xi$.

See [Th, D, L] for a detailed discussion of geodesic laminations and pinched models. 


\section{LOCAL PROPERTIES OF QUASISYMMETRIES OF $\mathcal{J}$}

Before we proceed, we briefly recall basic definitions and facts on quasiconformal and quasisymmetric maps. For more background one can consult [Vä, AIM, He].

A homeomorphism $f: U \rightarrow \tilde{U}$ between open regions in the plane $\mathbb{C}$ or the Riemann sphere $\widehat{\mathbb{C}}$ is called quasiconformal if $f$ is in the Sobolev space $W_{\text {loc }}^{1,2}$ and if there exists a constant $K \geq 1$ such that the (formal) Jacobi matrix $D f$ satisfies

$$
\|D f(z)\|^{2} \leq K \operatorname{det}(D f(z))
$$

for almost every $z \in U$. In this case we say $f$ is $K$-quasiconformal; the constant $K$ is called dilatation of $f$. The condition $f \in W_{\mathrm{loc}}^{1,2}$ means that the first distributional partial derivatives of $f$ are locally in $L^{2}$. A quasiconformal map is necessarily orientation preserving.

If $\left(X, d_{X}\right)$ and $\left(Y, d_{Y}\right)$ are metric spaces, a homeomorphism $f: X \rightarrow$ $Y$ is called quasisymmetric or a quasisymmetry if there exists a homeomorphism $\eta:[0, \infty) \rightarrow[0, \infty)$ such that

$$
\frac{d_{Y}(f(u), f(v))}{d_{Y}(f(u), f(w))} \leq \eta\left(\frac{d_{X}(u, v)}{d_{X}(u, w)}\right),
$$

for every triple of distinct points $u, v, w \in X$. If we want to emphasize the dependence on the distortion function $\eta$, we say that $f$ is $\eta$-quasisymmetric, or $\eta$-quasisymmetry.

Suppose $U$ and $V$ are subregions of $\widehat{\mathbb{C}}$. Then every orientationpreserving $\eta$-quasisymmetric homeomorphism $f: U \rightarrow V$ is $K$-quasiconformal with $K$ that depends only on $\eta$. Conversely, every properly normalized quasiconformal homeomorphism $f: U \rightarrow V$ is locally quasisymmetric, i.e., for every compact set $M \subset U$, the restriction $\left.f\right|_{M}: M \rightarrow f(M)$ is a quasisymmetry, quantitatively, i.e., $\eta$ depends only on $K$ and the relative distance between $M$ and $\partial U$; see AIM, p. 58, Theorem 3.4.1 and p. 71, Theorem 3.6.2] and [He, Theorem 11.14]. This is referred to as the egg yolk principle.

If we have a family of maps with the same dilatation or distortion function, then we say that the family is uniform. E.g., a family of homeomorphisms is uniformly quasisymmetric if there exists a homeomorphism $\eta:[0, \infty) \rightarrow[0, \infty)$ such that each map from the family is $\eta$-quasisymmetric. Inverses and compositions of quasiconformal or quasisymmetric maps are quantitatively quasiconformal or quasisymmetric, respectively.

According to the Ahlfors-Beurling theorem [BA], each orientation preserving $\eta$-quasisymmetric map $h: \mathbb{T} \rightarrow \mathbb{T}$ has a $K$-quasiconformal 
extension $H$ to the whole complex plane, where $K$ depends only on $\eta$. Conversely, the homeomorphic extension $h$ of each $K$-quasiconformal homeomorphism $H: \mathbb{D} \rightarrow \mathbb{D}$ or $H: \mathbb{C} \backslash \overline{\mathbb{D}} \rightarrow \mathbb{C} \backslash \overline{\mathbb{D}}$ to $\mathbb{T}$ is $\eta$-quasisymmetric for some $\eta$ that depends only on $K$.

Let $\xi$ be an orientation preserving homeomorphism of $\mathbb{C}$ that leaves $\mathcal{J}$ invariant. Let $U, V$ be bounded Fatou components of $f$ such that $\xi: U \rightarrow V$. Then the homeomorphism $\xi_{U, V}=\phi_{V} \circ \xi \circ \psi_{U}: \mathbb{T} \rightarrow \mathbb{T}$, where $\psi_{U}=\phi_{U}^{-1}$, preserves the set of dyadic points. Indeed, this follows from the fact that $\xi$ preserves the set of global cut-points of $\mathcal{J}$. By Lemma 3.4, the global homeomorphism $\xi$ also induces a homeomorphism $\xi_{\infty}$ of $\mathbb{T}$ that satisfies $\psi_{\infty} \circ \xi_{\infty}=\xi \circ \psi_{\infty}$. For the same reason as above, the induced map $\xi_{\infty}$ preserves the set $D_{\infty}=\cup_{n=0}^{\infty} D_{n}$, where $D_{n}$ is the set of all preimages of $\{1 / 3,2 / 3\}$ under $g^{n}$, with $g(z)=z^{2}$.

Let $\xi$ be a topologically extendable $\eta$-quasisymmetric self-map of $\mathcal{J}$. In what follows, we also denote by $\xi$ its homeomorphic extension to all of $\mathbb{C}$. Let $U$ and $V$ be bounded Fatou components of $f$ such that $\xi(U)=V$. In this case the maps $\xi_{U, V}$, where $U$ runs over all bounded Fatou components, are uniform quasisymmetries, i.e, they are $\eta^{\prime}$-quasisymmetries with $\eta^{\prime}$ that depends only on $\eta$. This follows from an elementary fact that all the bounded Fatou components are uniform quasidiscs, which, in turn, is a consequence of the hyperbolicity of $f$. In particular, each $\xi_{U, V}$ has a $K$-quasiconformal extension to $\mathbb{C}$, where $K$ depends only on $\eta$. The following lemma shows that the same holds for the $\operatorname{map} \xi_{\infty}$.

Lemma 4.1. Let $\xi$ be a topologically extendable $\eta$-quasisymmetric map of $\mathcal{J}$. Let $\xi_{\infty}$ be the induced map on the unit circle $\mathbb{T}$ that satisfies the semi-conjugation $\psi_{\infty} \circ \xi_{\infty}=\xi \circ \psi_{\infty}$. Then $\xi_{\infty}$ is an $\eta^{\prime}$-quasisymmetric homeomorphism of $\mathbb{T}$ with $\eta^{\prime}$ that depends only on $\eta$. In particular, $\xi_{\infty}$ has a $K$-quasiconformal extension to $\mathbb{C}$, where $K$ depends only on $\eta$.

Proof. It is enough to show that there exists $C>0$ that depends only on $\eta$, such that if $I$ and $J$ are two adjacent non-overlapping $\operatorname{arcs}$ on $\mathbb{T}$ that have the same lengths, then

$$
\operatorname{diam}\left(\xi_{\infty}(I)\right) / C \leq \operatorname{diam}\left(\xi_{\infty}(J)\right) \leq C \operatorname{diam}\left(\xi_{\infty}(I)\right) .
$$

To prove this, we first show that there exist constants $C_{1}, C_{1}^{\prime}>0$, such that if $I$ and $J$ are two adjacent non-overlapping arcs on $\mathbb{T}$, then

$$
\operatorname{diam}(I) / C_{1} \leq \operatorname{diam}(J) \leq C_{1} \operatorname{diam}(I)
$$

if and only if

$$
\operatorname{diam}\left(I^{\prime}\right) / C_{1}^{\prime} \leq \operatorname{diam}\left(J^{\prime}\right) \leq C_{1}^{\prime} \operatorname{diam}\left(I^{\prime}\right)
$$


where for an interval $I$ in $\mathbb{T}$ we denote by $I^{\prime}$ the corresponding Julia arc, i.e., $I^{\prime}=\psi_{\infty}(I)$; we use the same convention in the rest of this proof. In this statement, for the necessary part $C_{1}$ is given and $C_{1}^{\prime}$ depends only on $C_{1}$, and for the sufficiency part it is the other way around.

Indeed, since $\psi_{\infty}$ does not collapse arcs of $\mathbb{T}$ to points, it follows that if $I$ is such an $\operatorname{arc}$ with $\operatorname{diam}(I) \geq \epsilon>0$, then there exists $\delta>0$ that depends only on $\epsilon$, such that $\operatorname{diam}\left(I^{\prime}\right) \geq \delta$. Conversely, the uniform continuity of $\psi_{\infty}$ implies that if $\delta>0$ is given such that $\operatorname{diam}\left(I^{\prime}\right) \geq \delta$, then there exists $\epsilon>0$ that depends only on $\delta$, with $\operatorname{diam}(I) \geq \epsilon$.

Now, if $\operatorname{diam}(I) / C_{1} \leq \operatorname{diam}(J) \leq C_{1} \operatorname{diam}(I)$, then there exists a constant $\epsilon_{0}>0$ that depends only on $C_{1}$, such that for some $n \in \mathbb{N}_{0}$ we have

$$
\operatorname{diam}\left(I_{n}\right), \operatorname{diam}\left(J_{n}\right) \geq \epsilon_{0},
$$

where $I_{n}=g^{n}(I)$ and $J_{n}=g^{n}(J)$. From the previous paragraph it follows that (4.1) holds if and only if there exists $\delta_{0}>0$ such that

$$
\operatorname{diam}\left(I_{n}^{\prime}\right), \operatorname{diam}\left(J_{n}^{\prime}\right) \geq \delta_{0} .
$$

Here, for the "if" part, $\epsilon_{0}$ depends only on $\delta_{0}$, and for the "only if" part $\delta_{0}$ depends only on $\epsilon_{0}$. Thus, there exist constants $C_{2}, C_{2}^{\prime}>0$ that depend only on $\epsilon_{0}$ (or only on $\delta_{0}$ ), such that

$$
\operatorname{diam}\left(I_{n}\right) / C_{2} \leq \operatorname{diam}\left(J_{n}\right) \leq C_{2} \operatorname{diam}\left(I_{n}\right)
$$

and

$$
\operatorname{diam}\left(I_{n}^{\prime}\right) / C_{2}^{\prime} \leq \operatorname{diam}\left(J_{n}^{\prime}\right) \leq C_{2}^{\prime} \operatorname{diam}\left(I_{n}^{\prime}\right) .
$$

Note that $I_{n}^{\prime}=f^{n}\left(I^{\prime}\right)$ and $J_{n}^{\prime}=f^{n}\left(J^{\prime}\right)$. We may and will assume that $n$ is chosen not too large, so that $I_{n}^{\prime}$ and $J_{n}^{\prime}$ are properly contained in an open set where the appropriate branch of $f^{-n}$ is well-defined and conformal. The egg yolk principle now implies that there exists a constant $C_{1}^{\prime}>0$ that depends only on $C_{2}, C_{2}^{\prime}$, and hence only on $C_{1}$, such that

$$
\operatorname{diam}\left(I^{\prime}\right) / C_{1}^{\prime} \leq \operatorname{diam}\left(J^{\prime}\right) \leq C_{1}^{\prime} \operatorname{diam}\left(I^{\prime}\right) .
$$

The converse implication, namely that the last inequalities imply

$$
\operatorname{diam}(I) / C_{1} \leq \operatorname{diam}(J) \leq C_{1} \operatorname{diam}(I),
$$

for some $C_{1}>0$ that depends only on $C_{1}^{\prime}$, follows the same lines with the egg yolk principle applied to $f^{n}$ rather than to $f^{-n}$.

We are now ready to finish the proof. If $I$ and $J$ are two adjacent non-overlapping arcs of $\mathbb{T}$ that have the same lengths, then for some absolute constant $C_{1}^{\prime}>0$ we have

$$
\operatorname{diam}\left(I^{\prime}\right) / C_{1}^{\prime} \leq \operatorname{diam}\left(J^{\prime}\right) \leq C_{1}^{\prime} \operatorname{diam}\left(I^{\prime}\right) .
$$


Since $\xi$ is $\eta$-quasisymmetric, there exists a constant $C_{2}^{\prime}>0$ that depends only on $\eta$ and $C_{1}^{\prime}$, such that

$$
\operatorname{diam}\left(\xi\left(I^{\prime}\right)\right) / C_{2}^{\prime} \leq \operatorname{diam}\left(\xi\left(J^{\prime}\right)\right) \leq C_{2}^{\prime} \operatorname{diam}\left(\xi\left(I^{\prime}\right)\right)
$$

But, for each interval $I$ in $\mathbb{T}$, it follows from $\psi_{\infty} \circ \xi_{\infty}=\xi \circ \psi_{\infty}$ that $\xi\left(I^{\prime}\right)=\left(\xi_{\infty}(I)\right)^{\prime}$, and so

$$
\operatorname{diam}\left(\left(\xi_{\infty}(I)\right)^{\prime}\right) / C_{2}^{\prime} \leq \operatorname{diam}\left(\left(\xi_{\infty}(J)\right)^{\prime}\right) \leq C_{2}^{\prime} \operatorname{diam}\left(\left(\xi_{\infty}(I)\right)^{\prime}\right)
$$

Now we apply the above claim to conclude that there exists a constant $C>0$ that depends only on $C_{2}^{\prime}$ with

$$
\operatorname{diam}\left(\xi_{\infty}(I)\right) / C \leq \operatorname{diam}\left(\xi_{\infty}(J)\right) \leq C \operatorname{diam}\left(\xi_{\infty}(I)\right),
$$

and the lemma follows.

\section{ThOMPSON GROUP ACTION ON $\mathcal{J}$}

5.0.1. Thompson groups. The Thompson group $F$ is a group of orientation preserving piecewise linear homeomorphisms of the closed interval $[0,1]$ whose break points, i.e., points of non-differentiability, are dyadic points and such that on intervals of linearity the slopes are integer powers of 2. See CFP for background on the group $F$ as well as the Thompson group $T$ of the unit circle, defined below. It follows immediately that the elements of $F$ preserve the set of dyadic points. The group $F$ is generated by

$$
A(t)= \begin{cases}t / 2, & 0 \leq t \leq 1 / 2 \\ t-1 / 4, & 1 / 2 \leq t \leq 3 / 4 \\ 2 t-1, & 3 / 4 \leq t \leq 1\end{cases}
$$

and

$$
B(t)= \begin{cases}t, & 0 \leq t \leq 1 / 2, \\ t / 2+1 / 4, & 1 / 2 \leq t \leq 3 / 4 \\ t-1 / 8, & 3 / 4 \leq t \leq 7 / 8 \\ 2 t-1, & 7 / 8 \leq t \leq 1\end{cases}
$$

Similarly, the Thompson group $T$ is the group of orientation preserving piecewise linear (in the angular metric) homeomorphisms of the unit circle $\mathbb{T}$ that preserve the set of dyadic points, whose break points are dyadic points, and the slopes on intervals of linearity are integer powers of 2. Elements of the Thompson group $F$ induce in the obvious way elements of $T$ that fix $1 \in \mathbb{T}$. The group $T$ is generated 


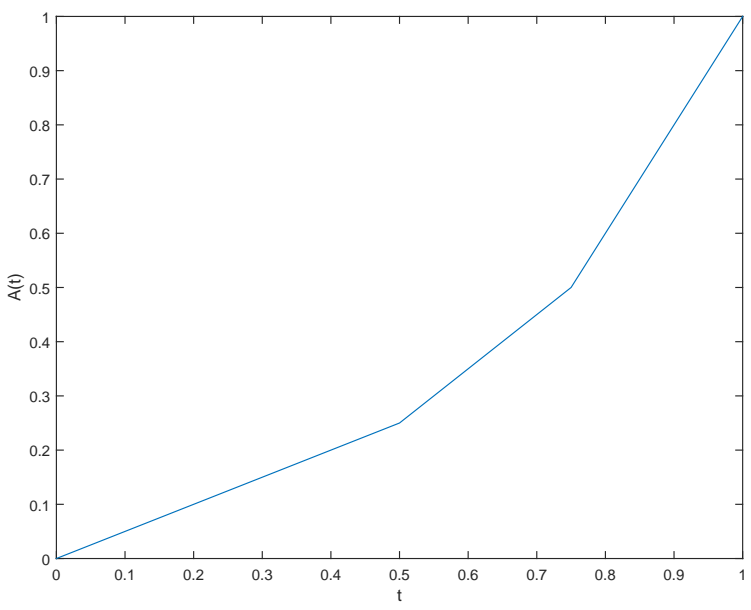

Figure 3. The graph of $A(t)$.

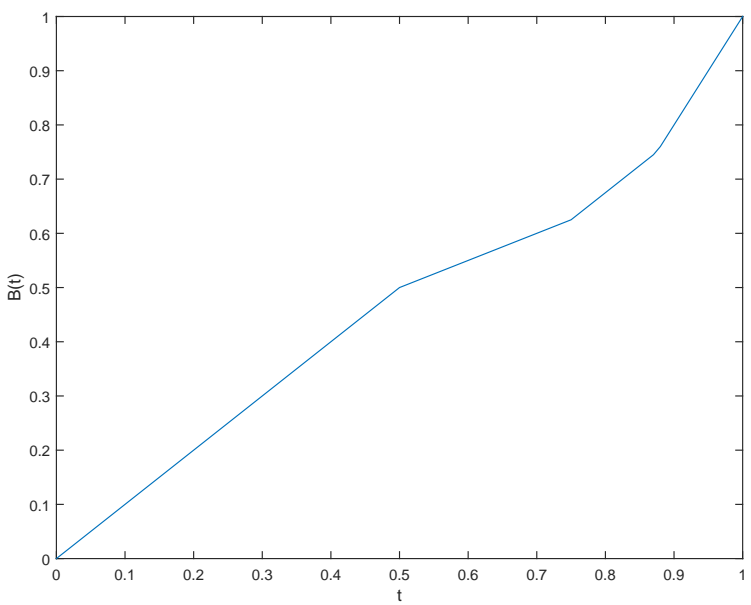

Figure 4. The graph of $B(t)$.

by elements $A, B, C$, where $A$ and $B$ are the homeomorphisms of $\mathbb{T}$ induced by the elements $A$ and $B$ of $F$, respectively, and

$$
C(t)= \begin{cases}t / 2+3 / 4, & 0 \leq t \leq 1 / 2 \\ 2 t-1, & 1 / 2 \leq t \leq 3 / 4 \\ t-1 / 4, & 3 / 4 \leq t \leq 1\end{cases}
$$

5.0.2. Pseudo-group $\Upsilon_{g}$. A pseudo-group $\Upsilon$ on a smooth manifold $M$ is a collection of diffeomorphisms $h: U_{h} \rightarrow V_{h}$ between open subsets of $M$ that satisfy the following axioms: 


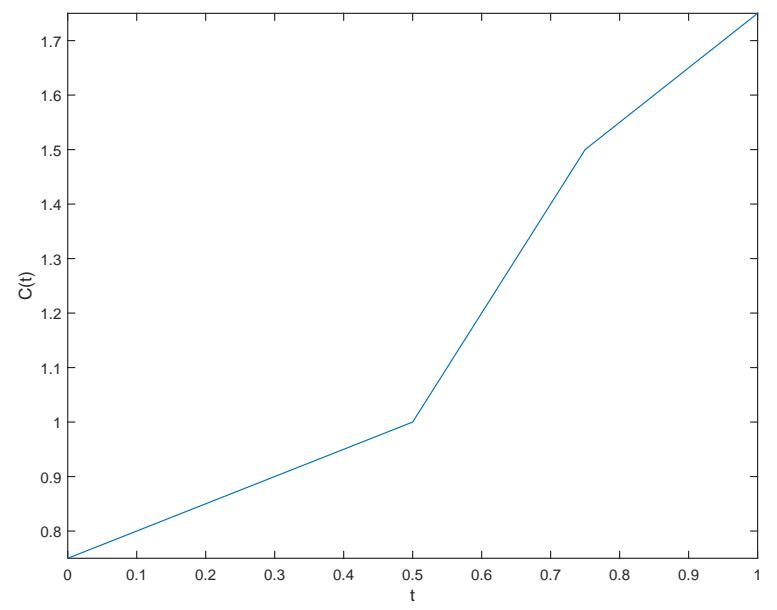

Figure 5. The graph of $C(t)$.

1. If $h_{1}$ and $h_{2}$ in $\Upsilon$ and $V_{h_{1}} \subseteq U_{h_{2}}$, then $h_{2} \circ h_{1} \in \Upsilon$;

2. If $h \in \Upsilon$, then $h^{-1} \in \Upsilon$;

3. $\operatorname{id}_{M} \in \Upsilon$;

4. If $h \in \Upsilon$ and $W \subseteq U_{h}$ is open, then the restriction $\left.h\right|_{W} \in \Upsilon$;

5. If $h: U_{h} \rightarrow V_{h}$ is a diffeomorphism between open sets in $M$, and if for each $p \in U_{h}$ there is an open neighborhood $W$ of $p$ in $U_{h}$, such that $\left.h\right|_{W} \in \Upsilon$, then $h \in \Upsilon$.

For examples and background on pseudo-groups the reader may consult, e.g., [CC, Ch. 2].

For further reference, let us formulate two elementary lemmas:

Lemma 5.1. Let $I_{0}$ and $I_{1}$ be two non-empty open arcs on $\mathbb{T}$ so that the endpoints are dyadic. Then there exists an orientation preserving piecewise linear map $\tau$ from $I_{0}$ onto $I_{1}$ whose break points are dyadic points and such that on each interval of linearity, the slope of $\tau$ in the angular metric is $2^{n}$ for some $n \in \mathbb{Z}$.

Proof. First, we may assume by applying rotations by dyadic numbers that both $I_{0}$ an $I_{1}$ have a common endpoint at 1 , and they can be identified with intervals in $[0,1]$ with one endpoint at 0 . Then the other endpoints of $I_{0}$ and $I_{1}$ are dyadic points $d_{0}, d_{1} \in(0,1]$, respectively. We may assume that $d_{0}=k / 2^{n}, d_{1}=m / 2^{n}$ and $k<m$. We now apply the identity to the first $k-1$ intervals of length $1 / 2^{n}$ comprising $\left(0, d_{0}\right]$, and scale the last such interval by 2 . All the slopes of such a map on $\left(0, d_{0}\right]$ are integer powers of 2 and this map takes $\left(0, d_{0}\right]$ to the interval $\left(0, d_{0}+1 / 2^{n}\right]$. Inductive process now finishes the proof. 
Recall that $g$ stands for the doubling map of the circle. Given a path $\gamma:[0,1] \rightarrow \mathbb{T}$, a moment $m \in \mathbb{N}_{0}$, and a preimage $z_{0} \in g^{-m}(\gamma(0))$, we can uniquely lift the path $\gamma$ by $g^{m}$ to a path $\delta:[0,1] \rightarrow \mathbb{T}$ with $\delta(0)=$ $z_{0}$. Informally, we will refer to the corresponding analytic continuation $\delta(t)=g^{-m}(\gamma(t))$ of $g^{-m}$ along $\gamma$ as the branch of $g^{-m}$ on $\gamma$ that starts at $z_{0}$.

In particular, we can consider an arc $I \subset \mathbb{T}$, a curve $g^{n}: I \rightarrow \mathbb{T}$, and a branch of some $g^{-m}$ on this curve. We obtain a composition $\xi=g^{-m} \circ g^{n}$ on $I$. If the image $\tilde{I}:=\xi(I)$ is also an arc on $\mathbb{T}$ (i.e., $\xi: I \rightarrow \mathbb{T}$ does not "wrap around the circle"), then $\xi: I \rightarrow \tilde{I}$ is a linear (in the angular coordinate) diffeomorphism with slope $2^{n-m}$. Let us denote the pseudo-group of such diffeomorphisms by $\Upsilon_{g}$.

Lemma 5.2. If $I$ and $\tilde{I}$ are arcs of $\mathbb{T}$ whose endpoints are dyadic, and if $\xi$ is a linear map of $I$ onto $\tilde{I}$ such that the slope of $\xi$ in the angular coordinate is an integer power of 2 , then $\xi$ belongs to the pseudo-group $\Upsilon_{g}$.

Proof. Let $I=[a, b]$ and $\tilde{I}=[\tilde{a}, \tilde{b}]$. Since $a$ is assumed to be dyadic, we can find $n \in \mathbb{N}_{0}$ such that $g^{n}(a)=1$. Let us consider the branch of $g^{-n}$ on the path $g^{n}: I \rightarrow \mathbb{T}$ that starts at 1 . The composition $r=g^{-n} \circ g^{n}: I \rightarrow \mathbb{T}$ is a linear (in the angular coordinate) map with slope 1 . Hence it rotates $I$ to a dyadic interval $J=[1, e(\theta)], \theta \in[0,1]$.

Likewise, we can find a moment $\tilde{n} \in \mathbb{N}_{0}$ and a branch of $g^{-\tilde{n}}$ such that the composition $\tilde{r}=g^{-\tilde{n}} \circ g^{\tilde{n}}: \tilde{I} \rightarrow \mathbb{T}$ rotates $\tilde{I}$ to a dyadic interval $\tilde{J}=[1, e(\tilde{\theta})], \tilde{\theta} \in[0,1]$. Moreover, $\tilde{\theta}=2^{k} \theta$, where $2^{k}$ is the slope of $\xi$ on $I, k \in \mathbb{Z}$. Hence $\tilde{J}=g^{k}(J)$, where, in the case when $k$ is negative, the branch of $g^{k}$ is chosen so that the point 1 is fixed.

The desired map can now be written as $\tilde{r}^{-1} \circ g^{k} \circ r$.

Let us say that a piecewise linear homeomorphism $\mathbb{T} \rightarrow \mathbb{T}$ is piecewise dynamical if its restriction to every interval of linearity belongs to the pseudo-group $\Upsilon_{g}$.

Corollary 5.3. Any element of the Thompson group $T$ is piecewise dynamical.

For instance, the $A$-generator of the Thompson group can be obtained as follows:

$$
A=\left\{\begin{array}{ll}
g^{-1}, & 0 \leq t \leq 1 / 2, \quad \text { where the branch } g^{-1} \text { fixes } 1 \\
g^{-2} \circ g^{2}, & 1 / 2 \leq t \leq 3 / 4, \\
g, & 3 / 4 \leq t \leq 1
\end{array} \quad \text { where } g^{-2}:(0,1) \rightarrow(1 / 4,1 / 2),\right.
$$


5.0.3. Piecewise dynamical action of the Thompson group on $\mathcal{J}$. Similarly to $\Upsilon_{g}$, let us consider the dynamical pseudo-group $\Upsilon_{f}$ comprising local isomorphisms $\psi=f^{-m} \circ f^{n}: V \rightarrow W$, where $m, n \in \mathbb{N}_{0}, V$ is simply connected, $\left.f^{n}\right|_{V}$ is univalent, and $\left.f^{-m} \circ f^{n}\right|_{V}$ is a well defined analytic branch of the algebraic function $f^{-m} \circ f^{n}$. Note that in the $\log$-Böttcher coordinate $u=\log \phi_{\infty}(z)$, the map $\psi$ is affine with slope $2^{n-m}, u \mapsto 2^{n-m} u+c, c \in \mathbb{R}$.

Let us say that a homeomorphism $\xi: J \rightarrow \tilde{J}$ between Julia arcs is piecewise dynamical if $J$ can be decomposed into finitely many Julia $\operatorname{arcs} J_{i}$ that share only global cut-points, so that each restriction $\left.\xi\right|_{J_{i}}$, extended to some neighborhood $W_{i}$, belongs to the pseudo-group $\Upsilon_{f}$. If $J=\tilde{J}=\mathcal{J}$, piecewise dynamical homeomorphisms $\xi: \mathcal{J} \rightarrow \mathcal{J}$ form a group, and we denote it by $\operatorname{Dyn}(\mathcal{J})$.

Let us also say that a map $h: U \rightarrow V$ between two bounded Fatou components respects the Böttcher coordinate if it is the identity in the Böttcher coordinates of these components, i.e.,

$$
\phi_{V} \circ h \circ \psi_{U}=\mathrm{id}: \mathbb{D} \rightarrow \mathbb{D} \text {. }
$$

Lemma 5.4. The Thompson group $T$ induces the group of piecewise dynamical quasisymmetries of $\mathcal{J}$ that keep the central Fatou component $\partial U_{0}$ invariant. Moreover, they admit global quasiconformal extensions to $\mathbb{C}$ that respect the Böttcher coordinates of all non-central bounded Fatou components.

Proof. Let $\xi_{0}$ be an element of $T$ acting on $\partial U_{0}$ piecewise linearly in the (inner) angular coordinate. We want to extend it to an orientation preserving quasisymmetric homeomorphism of $\mathcal{J}$ (actually, to a global quasiconformal map of $(\mathbb{C}, \mathcal{J})$ ).

Let $I \subseteq \partial U_{0}$ be an arc of linearity for $\xi_{0}$. By Corollary $5.3,\left.\xi_{0}\right|_{I}=$ $f^{-m} \circ f^{n}$ for some $n, m \in \mathbb{N}_{0}$. Hence $\xi_{0}$ admits an analytic extension $\hat{\xi}_{0}$ to a neighborhood $V$ of $I$ as an element of the dynamical pseudo-group $\Upsilon_{f}$.

Let us puncture out from $I$ all dyadic points of level $\leq n$. Take any complementary (open) interval $J \subseteq I$. Let $\Omega_{J}$ be the region bounded by $J$ and two external rays landing at $\partial J$ (where the rays are selected so that $\Omega_{J}$ does not intersect the wakes attached to $\left.\partial J\right)$. Since $f^{n}\left(\Omega_{J}\right)$ is disjoint from $U_{0} \cup U_{-1}$, all branches of $f^{-m}$ are well defined on it, so $\hat{\xi}_{0}$ extends analytically to $\Omega_{J}$ (mapping it to some $\Omega_{\tilde{J}}$ ). We restrict this map to the intersection of $\Omega_{J}$ with the filled Julia set $\mathcal{K}$ and extend it continuously to the closure $\mathcal{K}_{J}$ of this set.

In this way, we extend $\xi_{0}$ to all $\mathcal{K} \backslash U_{0}$, except finitely many limbs attached to $\partial U_{0}$. Let $S_{z}$ be one of the wakes containing such a limb. 
Its root $z \in \partial U_{0}$ is a dyadic point of some level $k$. Let $\tilde{z}=\xi_{0}(z)$. Since $\xi_{0}$ is Thompson, $\tilde{z}$ is also dyadic (of some level $\tilde{k}$ ), so there is a wake $S_{\tilde{z}}$ rooted at $\tilde{z}$.

Recall that $S_{0}$ is the sector bounded by $\rho^{ \pm \frac{1}{3}}$ that contains $U_{0}$. Then $f^{k}$ and $f^{\tilde{k}}$ conformally map $S_{z}$ and $S_{\tilde{z}}$, respectively, onto $S_{0}$. (If $k$ or $\tilde{k}$ is 0 , then $f$ takes $S_{z}$, respectively $S_{\tilde{z}}$, to $S_{0}$.)

Thus, we obtain a map $\xi_{z}=f^{-\tilde{k}} \circ f^{k}: S \rightarrow \tilde{S}$ of pseudo-group $\Upsilon_{f}$. Restricting it to the filled Julia set, we obtain a homeomorphism $\xi_{z}: \mathcal{K}_{z} \rightarrow \mathcal{K}_{\tilde{z}}$ between the corresponding limbs. Putting these maps together, we obtain an extension of $\xi_{0}$ to a homeomorphism $\xi: \mathcal{K} \backslash U_{0} \rightarrow$ $\mathcal{K} \backslash U_{0}$ that restricts to an element in $\operatorname{Dyn}(\mathcal{J})$.

Let us show that the map $\xi$ admits a global quasiconformal extension to the complex plane. By definition, it has a conformal extension to each non-central bounded Fatou component as a conformal map $f^{-m}$ 。 $f^{n}$. Since $\partial U_{0}$ is a quasicircle and $\xi_{0}: \partial U_{0} \rightarrow \partial U_{0}$ is piecewise linear in the inner angular coordinate, it is quasisymmetric. Hence it admits a quasiconformal extension to $U_{0}$ (Ahlfors-Beurling extension [BA]).

We now extend $\xi$ to $U_{\infty}$. Let $J_{i}$ be the arcs of $\partial U_{0}$ considered above, and let $z_{i}$ be their boundary dyadic points. Let $\mathcal{Y}_{i}=\partial \mathcal{K}_{J_{i}}$ and $\mathcal{Z}_{i}=$ $\partial \mathcal{K}_{z_{i}}$ be the corresponding Julia arcs. In the Böttcher coordinate, these Julia arcs correspond to some $\operatorname{arcs} Y_{i}$ and $Z_{i}$ tessellating the circle $\mathbb{T}$.

Since $\xi$ belongs to the dynamical pseudo-group $\Upsilon_{f}$ on each $\mathcal{Y}_{i}$ and $\mathcal{Z}_{i}$, it induces linear (in the outer angular coordinate of the circle $\mathbb{T}$ ) maps $Y_{i} \rightarrow \tilde{Y}_{i}$ and $Z_{i} \rightarrow \tilde{Z}_{i}$. Moreover, since $\xi_{0}$ preserves the cyclic order in which the arcs $J_{i}$ and points $z_{i}$ appear on $\partial U_{0}$, these maps form a single piecewise linear homeomorphism $h: \mathbb{T} \rightarrow \mathbb{T}$. Applying the Ahlfors-Beurling extension once again, we obtain a quasiconformal extension $\hat{h}$ of $h$ to $U_{\infty}$.

Note that $h$ preserves the basilica lamination on $\mathbb{T}$. It is so on

$$
\mathbb{T} \backslash \bigcup \partial Z_{i} \quad\left(=\mathbb{T} \backslash \bigcup \partial Y_{i}\right)
$$

since $\xi$ admits local homeomorphic extensions to $\mathbb{C}$ near any point of $\mathcal{J} \backslash\left\{z_{i}\right\}$. It is also true at the points of $\bigcup \partial Z_{i}$. Indeed, the lamination pairs the boundary points of each $Z_{i}$ (corresponding to the rays enclosing the $\left.\operatorname{limb} \mathcal{Z}_{i}\right)$. Since $\xi$ maps the $\operatorname{limb} \mathcal{Z}_{i}$ to another limb $\tilde{\mathcal{Z}}_{i}$, the boundary points of $\tilde{Z}_{i}=h\left(Z_{i}\right)$ correspond to the rays enclosing $\tilde{\mathcal{Z}}_{i}$. So, they are paired by the lamination. Hence $\hat{h}$ descends to a homeomorphism of $(\mathbb{C}, \mathcal{K})$ providing us with the desired quasiconformal extension of $\xi$ to $\mathbb{C}$. Here we also need the fact that the Julia set $\mathcal{J}$ is removable for quasiconformal maps [Jo, Corollary 2 on p. 5 and Remark on p. 3]. We continue to denote this extension by $\xi$. 
It remains to show that $\xi$ respects the Böttcher coordinates of all non-central bounded Fatou components. In each such component $U$ it has the form $f^{-m} \circ f^{n}$ and maps it to another such component $\tilde{U}$. The claim now follows from the fact that the Böttcher coordinate of a bounded Fatou component with dynamical distance $k$ to $U_{0}$ is $\phi_{0} \circ f^{k}$. Indeed, since in the construction above $n$ is chosen so that $f^{n}(U)$ does not contain the postcritical points, we have that $n$ is at most the dynamical distance from $U$ to $U_{0}$. If $l \in \mathbb{N}_{0}$ is such that $n+l$ is the dynamical distance from $U$ to $U_{0}$, then for the branch of $f^{-l}$ that takes $U_{0}$ to $f^{n}(U)$, the map $\xi$ in $U$ is given by $f^{-m} \circ f^{-l} \circ f^{n+l}$. We conclude that $m+l$ is the dynamical distance from $\tilde{U}$ to $U_{0}$ and for an appropriate branch of $f^{-(m+l)}$ the map $\xi$ in $U$ is given by

$$
f^{-(m+l)} \circ f^{n+l} \text {. }
$$

This is equivalent to saying that $\xi$ respects the Böttcher coordinates.

5.0.4. Thompson-like action. The Thompson group $T$ is based on dyadic points, iterated preimages of the fixed point 1 under the doubling map $g$. More generally, one can consider iterated preimages of any periodic cycle $\boldsymbol{\alpha}$ and define the associated Thompson-like group $T_{\boldsymbol{\alpha}}$. I.e., the elements of $T_{\boldsymbol{\alpha}}$ preserve the set $D_{\boldsymbol{\alpha}}$ of all preimages of the elements of $\boldsymbol{\alpha}$ under the iterates of the map $g$, the break points are at $D_{\boldsymbol{\alpha}}$, and the slopes are integer powers of 2 . The above discussion readily extends to this setting, and in particular, we have the following counterpart of Corollary 5.3 .

Corollary 5.5. Any element of the Thompson-like group $T_{\boldsymbol{\alpha}}$ is piecewise dynamical.

In what follows, we let $\boldsymbol{\alpha}=\{1 / 3,2 / 3\}$. Let $T_{\boldsymbol{\alpha}}^{b}$ be the subgroup of the Thompson-like group $T_{\boldsymbol{\alpha}}$ consisting of homeomorphisms $\mathbb{T} \rightarrow \mathbb{T}$ that preserve the basilica lamination.

Lemma 5.6. Any piecewise dynamical homeomorphism $\xi: \mathcal{J} \rightarrow \mathcal{J}$ induces a homeomorphism $\xi_{\infty}: \mathbb{T} \rightarrow \mathbb{T}$ that belongs to $T_{\boldsymbol{\alpha}}^{b}$. Moreover, the map $\operatorname{Dyn}(\mathcal{J}) \rightarrow T_{\boldsymbol{\alpha}}^{b}, \xi \mapsto \xi_{\infty}$, is a group isomorphism.

Proof. It follows from the definition that any piecewise dynamical homeomorphism $\xi$ of $\mathcal{J}$ has a homeomorphic extension to $\overline{U_{\infty}}$, and therefore, by Lemma 3.4, the induced homeomorphism $\xi_{\infty}$ of $\mathbb{T}$ preserves the basilica lamination. The rest of the properties of $\xi_{\infty}$ follow from the assumption that $\xi$ is piecewise dynamical. 
Conversely, any such map $\xi_{\infty}$ extends to a homeomorphism of $\mathbb{C} \backslash \mathbb{D}$ and hence descends to a homeomorphism $\xi$ of $\mathcal{J}$ by Lemma 3.4. The homeomorphism $\xi$ has to be piecewise dynamical because such is $\xi_{\infty}$ according to Corollary 5.5 .

The statement about the map $\xi \mapsto \xi_{\infty}$ being an isomorphism follows immediately from the relation

$$
\psi_{\infty} \circ \xi_{\infty}=\xi \circ \psi_{\infty}
$$

One element of $T$ that will be useful in what follows is the rotation $\rho(z)=-z$. It is given by $f^{-2} \circ f^{2}$, where the branch of $f^{-2}$ is chosen so that $f^{-2}(\alpha)=-\alpha$.

\section{INVERSION OF $\mathcal{J}$}

In this section we define an inversion $\iota$ of $\mathcal{J}$ that along with the action of the Thompson group $T$ on $\mathcal{J}$ generates a group whose elements approximate every quasisymmetric self-map of $\mathcal{J}$ quantitatively. We recall that $\mathcal{J}_{\alpha}=\partial \mathcal{K}_{\alpha}$, where $\mathcal{K}_{\alpha}$ is the limb rooted at $\alpha$ that contains the boundary $\partial U_{-1}$ of the bounded Fatou component $U_{-1}$ containing -1. Also, $\mathcal{J}_{0}=\left(\mathcal{J} \backslash \mathcal{J}_{\alpha}\right) \cup\{\alpha\}$.

Lemma 6.1. There exists a quasisymmetric homeomorphism $\iota$ of $\mathcal{J}$ that fixes $\alpha$, interchanges $\mathcal{J}_{\alpha}$ and $\mathcal{J}_{0}$, and satisfies

$$
\iota^{2}=\mathrm{id} \text {. }
$$

Moreover, ¿ has a global quasiconformal extension to $\mathbb{C}$ and respects the Böttcher coordinates of all bounded Fatou components.

Proof. We define $\iota$ by $\iota=f$ on $\mathcal{J}_{\alpha}$, and $\iota=f^{-1}$ on $\mathcal{J}_{0}$. Here we choose the branch of $f^{-1}$ that fixes $\alpha$. We prove that so defined $\iota$ is quasisymmetric by showing that it extends to a quasiconformal map of $\mathbb{C}$.

In each bounded component of the Fatou set whose closure intersects $\mathcal{J}_{\alpha}$ we extend $\iota$ by $\iota=f$, and in each such component whose closure intersects $\mathcal{J}_{0}$ we extend it by $\iota=f^{-1}$. In particular, it is immediate that if $U$ is any bounded Fatou component and $\partial \tilde{U}=\iota(\partial U)$, then this extension of $\iota$ respects the Böttcher coordinates of $U$ and $\tilde{U}$.

The map $\iota$ has obvious conformal extensions to both wakes in $\mathbb{C}$ bounded by the two external rays in $U_{\infty}$ landing at $\alpha$. These extensions are conjugate by $\phi_{\infty}$ to the maps $g(z)=z^{2}$ for $1 / 3 \leq e(\theta) \leq 2 / 3$ and $z \mapsto-\sqrt{z}$ for $-1 / 3 \leq e(\theta) \leq 1 / 3$, respectively, where the principal branch of the square root is selected. We can use the Ahlfors-Beurling extension $[\mathrm{BA}]$ to extend this piecewise linear map to a quasiconformal 
map of $\mathbb{C} \backslash \overline{\mathbb{D}}$ onto itself. This quasiconformal extension is conjugated back by $\phi_{\infty}$ to a quasiconformal map of $U_{\infty}$ onto itself that agrees with $\iota$ on the boundary of $U_{\infty}$.

Combining the above quasiconformal extension of $\iota$ with the conformal extensions into bounded Fatou components, we obtain a homeomorphism of $\mathbb{C}$ that is quasiconformal on the Fatou set. But the Julia set of a postcritically finite polynomial is removable for quasiconformal maps [Jo], and so we get a quasiconformal map of $\mathbb{C}$. Such a map is quasisymmetric, and hence $\iota$ is quasisymmetric on $\mathcal{J}$.

We call the homeomorphism $\iota$ the inversion with respect to $\alpha$. The composition $\sigma=\rho \circ \iota$ with the rotation $\rho$, given by $\rho(z)=-z$, acts as a shift to the right by one on the infinite chain $\mathcal{C}$ of bounded Fatou components that intersect the real line. This follows from the fact that $\sigma$ preserves $\mathcal{C}$ and takes $\partial U_{-1}$ to $\partial U_{0}$. Moreover, $\sigma$ respects the Böttcher coordinates of all bounded Fatou components, except that $\sigma: U_{-1} \rightarrow U_{0}$ is $180^{\circ}$-rotation in the Böttcher coordinates. (In general, we will say that a map $h: U \rightarrow V$ between bounded Fatou components rotates the Böttcher coordinate if $\phi_{U} \circ h \circ \psi_{V}$ is a rotation of the disk D.)

These remarks allow us to derive the following property:

Lemma 6.2. The group $\hat{T}$ generated by the Thompson group $T$ and $\iota$ acts transitively on the vertices of $\mathfrak{T}$. Moreover, let $U$ be an arbitrary bounded Fatou component, and let $p$ be the shortest combinatorial chain in $\mathfrak{T}$ that joins $U_{0}$ to $U$. Then there exists $t \in \hat{T}$ with the following properties:

(i) $t(U)=U_{0}$ and $t$ respects the Böttcher coordinate;

(ii) for any bounded Fatou component $V \notin p$, the map $t: V \rightarrow t(V)$ respects the Böttcher coordinate;

(iii) for any bounded Fatou component $V \in p$, the map $t: V \rightarrow t(V)$ rotates the Böttcher coordinate by some dyadic angle.

Proof. Suppose that $p=\left\{U_{0}, U_{1}, \ldots, U_{k}=U\right\}$. We will argue by induction on $k$. The base $k=0$ is obvious. In general, the component $U_{1}$ touches $U_{0}$, and so the base point of $U_{1}$ corresponds to a dyadic point on $\partial U_{0}$. The group $T$ contains rotations by any dyadic angle, and hence acts transitively on the dyadic points of $\partial U_{0}$. Thus there exists an element $t_{1} \in T$ such that $t_{1}\left(U_{1}\right)=U_{-1}$. The elements of $T$ preserve the combinatorial distance on $\mathfrak{T}$, and therefore $t_{1}(U)$ has the same combinatorial distance $k$ to $U_{0}$ as $U$. Now we apply the shift $\sigma$ to take $U_{-1}$ to $U_{0}$. The combinatorial distance from $\sigma \circ t_{1}(U)$ to $U_{0}$ is 
$k-1$, and we can apply the inductive hypothesis. Let $t_{2} \in \hat{T}$ be an element given by the inductive hypothesis that takes $\sigma \circ t_{1}(U)$ to $U_{0}$.

The map $t^{\prime}=t_{2} \circ \sigma \circ t_{1} \in \hat{T}$ takes $U$ to $U_{0}$. Moreover, $\sigma \circ t_{1}$ respects the Böttcher coordinates of all non-central bounded Fatou components except $U_{1}$, on which it rotates the coordinate by $180^{\circ}$. On $U_{0}$ itself, it rotates the Böttcher coordinate by some dyadic angle. By the inductive assumption, $t^{\prime} \in \hat{T}$ respects the Böttcher coordinate for all $V \notin p$ and rotates it for all $V \in p$ by some dyadic angles. Finally, by postcomposing $t^{\prime}$ with a rotation $\gamma$ in $T$, we can ensure that $t=\gamma \circ t^{\prime}: U \rightarrow U_{0}$ respects the Böttcher coordinate as well.

\section{Proof of Theorem 1.1}

Let $n \in N$ be arbitrary and let $\Pi_{n}=\left\{J_{k}, k=1,2, \ldots, 2^{n+1}\right\}$ be the partition of $\mathcal{J}$ by Julia arcs described in Section 3. We replace the map $\xi$ restricted to each $J_{k}$ by a piecewise dynamical map as follows.

Lemma 7.1. Let $\xi$ be a topologically extendable $\eta$-quasisymmetric map of $\mathcal{J}$. Then there exist $N \in \mathbb{N}_{0}$ and a finite family $\mathcal{F}$ of Julia arcs that depend only on $\eta$ and have the following property. For all $n \geq N$, for any $J \in \Pi_{n}$, if $\tilde{J}=\xi(J)$, then there exists $M \in \mathbb{N}_{0}$ with $\tilde{\Lambda}=f^{M}(\tilde{J}) \in$ $\mathcal{F}$. Moreover, $\tilde{\Lambda}$ is the closure of a connected component of $\mathcal{J} \backslash\left\{\tilde{z}_{1}, \tilde{z}_{2}\right\}$ for some global cut-points $\tilde{z}_{1}, \tilde{z}_{2} \in \partial U_{0}$, and $f^{-M}$ is conformal in a neighborhood of $\tilde{\Lambda}$.

Proof. We first note that $\xi$ has a $K$-quasiconformal extension to $\mathbb{C}$, where $K$ depends only on $\eta$. Indeed, it follows from Lemma 4.1 that the homeomorphism $\xi_{\infty}$ of $\mathbb{T}$ defined by

$$
\psi_{\infty} \circ \xi_{\infty}=\xi \circ \psi_{\infty}
$$

has a $K$-quasiconformal extension to $\mathbb{C} \backslash \overline{\mathbb{D}}$, where $K$ depends only on $\eta$. Since $\phi_{\infty}$ is conformal in $U_{\infty}$, it implies that $\xi$ has an extension to $U_{\infty}$ as a $K$-quasiconformal homeomorphism. Likewise, the discussion preceding Lemma 4.1 implies that $\xi$ has $K$-quasiconformal extension to each bounded Fatou component, perhaps with a different $K$ but that depends only on $\eta$. By [Jo], the Julia set $\mathcal{J}$ is removable for quasiconformal maps, and therefore the claim follows.

Assume that the Julia arc $J$ in the statement of the lemma is determined by adjacent external rays $\rho_{1}, \rho_{2} \in R_{n}$ with landing points $z_{1}, z_{2}$, respectively. There are two cases to consider: $z_{1}=z_{2}$ and $z_{1} \neq z_{2}$.

If $z=z_{1}=z_{2}$, then $z$ is the root of a bounded Fatou component $U$ such that $\partial U \subseteq J$, and $J=J_{z}=\partial \mathcal{K}_{z}$, where $\mathcal{K}_{z}$ is the limb rooted at $z$. Since any homeomorphism of $\mathcal{J}$ preserves the set of global cut-points, 
$\xi(z)$ is such a point. Moreover, from [He, Proposition 10.8] applied to the quasisymmetry $\xi$ it follows that there exists $N \in \mathbb{N}_{0}$ that depends only on $K$ such that if $n \geq N$, then $J \in \Pi_{n}$ implies that the Julia arc $\tilde{J}$ does not contain $\partial U_{0}$. Roughly speaking, [He, Proposition 10.8] states that if $A$ and $B$ are overlapping sets in a metric space with the diameter of $A$ being smaller than the diameter of $B$, then an application of a quasisymmetric map with controlled distortion cannot spoil this relationship between the diameters of the corresponding images of $A$ and $B$ too much, quantitatively. In particular, it follows from the proof of Lemma 2.1 that $\xi(z)$ is the root of the bounded Fatou component $\tilde{U}$ such that $\partial \vec{U}=\xi(\partial U)$. Let $M \in \mathbb{N}_{0}$ be the dynamical distance from $\tilde{U}$ to $U_{0}$. Then $\tilde{\Lambda}=f^{M}(\tilde{J})=\mathcal{J}_{0}$, and the claim follows in this case, with $\mathcal{F}$ consisting of the single element $\mathcal{J}_{0}$. We have $\tilde{z}_{1}=\tilde{z}_{2}=\alpha$. The fact that $f^{-M}$ is conformal in a neighborhood of $\mathcal{J}_{0}$ follows from the assumption that $M$ is the dynamical distance from $\tilde{U}$ to $U_{0}$.

Now assume that $z_{1} \neq z_{2}$. We know from Lemma 3.1 that there exists a bounded Fatou component $U$ such that $z_{1}, z_{2} \in \partial U$. As above, there exists $N \in \mathbb{N}_{0}$ that depends only on $K$ such that if $n \geq N$, then for $J \in \Pi_{n}$ we have that $\tilde{J}$ does not contain $\partial U_{0}$. Let $\mathcal{J}_{z_{1}}=\partial \mathcal{K}_{z_{1}}$ and $\mathcal{J}_{z_{2}}=\mathcal{K}_{z_{2}}$, where $\mathcal{K}_{z_{1}}, \mathcal{K}_{z_{2}}$ are the two limbs that are attached to $J$ at $z_{1}$ and $z_{2}$, respectively.

The egg yolk principle applied to an appropriate branch of $f^{-n}$ implies that $\operatorname{diam} \mathcal{J}_{z_{i}} \geq c \operatorname{diam} J, i=1,2$, for some $c>0$ that does not depend on $n$ or $J$. Let $\tilde{\mathcal{J}}_{i}=\xi\left(\mathcal{J}_{z_{i}}\right), i=1,2$. Since $\xi$ is quasisymmetric, an application of [He, Proposition 10.8] gives that $\operatorname{diam} \tilde{\mathcal{J}}_{i} \geq$ $\tilde{c} \operatorname{diam} \tilde{J}, i=1,2$, where the constant $\tilde{c}$ depends only on $K$. Since $f$ is hyperbolic, there exist constants $C=C(f)>0$ and $M \in \mathbb{N}_{0}$ with the following properties. First, for $\tilde{\Lambda}=f^{M}(\tilde{J})$ we have $\operatorname{diam}(\tilde{\Lambda}) \geq C$. Then, there exist a point $p \in \tilde{\Lambda}$ and $r>0$, such that $\tilde{\Lambda}$ is contained in the disc $B(p, r / 4)$. Finally, the disc $B(p, r)$ does not contain 0 and 1 , i.e., the postcritical points of $f$. In particular, $r \geq 2 C$. Since there are only finitely many large limbs of $\mathcal{J}$, the claim about the existence of a finite family $\mathcal{F}$ would follow if we show that there is a lower bound depending only on $K$ for $\operatorname{diam} f^{M}\left(\tilde{\mathcal{J}}_{i}\right), i=1,2$. If both $f^{M}\left(\tilde{\mathcal{J}}_{1}\right)$ and $f^{M}\left(\tilde{\mathcal{J}}_{2}\right)$, are not contained in $B(p, r / 2)$, then

$$
\operatorname{diam} f^{M}\left(\tilde{\mathcal{J}}_{i}\right) \geq r / 4 \geq C / 2, i=1,2
$$

If $f^{M}\left(\tilde{\mathcal{J}}_{i}\right)$ is contained in $B(p, r / 2)$ for some $i=1,2$, then the claim follows from the egg yolk principle applied to $f^{-M}$ in the disc $B(p, r)$, and the fact that $\operatorname{diam} \tilde{\mathcal{J}}_{i} \geq \tilde{c} \operatorname{diam} \tilde{J}, i=1,2$. 
The claim that, in the case $z_{1} \neq z_{2}$, the map $f^{-M}$ is conformal in a neighborhood of $\tilde{\Lambda}$ follows from the assumption that $\tilde{\Lambda}$ is contained in $B(p, r / 4)$ and $B(p, r)$ does not contain either 0 or 1 .

Finally, $\tilde{\Lambda}$ is the closure of a connected component of $\mathcal{J} \backslash\left\{\tilde{z}_{1}, \tilde{z}_{2}\right\}$, where $\tilde{z}_{1}=f^{M}\left(\xi\left(z_{1}\right)\right), \tilde{z}_{2}=f^{M}\left(\xi\left(z_{2}\right)\right)$, and these points belong to the boundary of a bounded Fatou component $V$. The component $V$ does not have to be $U_{0}$. However, $\operatorname{since} \operatorname{diam}(\tilde{\Lambda}) \geq C=C(f)$, the dynamical distance $m \in \mathbb{N}_{0}$ from $V$ to $U_{0}$ depends only on $C$. The set $f^{m}(\tilde{\Lambda})$ is a connected component of $\mathcal{J} \backslash\left\{f^{m}\left(\tilde{z}_{1}\right), f^{m}\left(\tilde{z}_{2}\right)\right\}$, and $f^{m}\left(\tilde{z}_{1}\right), f^{m}\left(\tilde{z}_{2}\right)$ belong to $\partial U_{0}$. Moreover, if we replace each element $\tilde{\Lambda} \in \mathcal{F}$ by $f^{m}(\tilde{\Lambda})$, all the other properties will be unchanged since $m$ depends only on $C$, and hence only on $f$. This completes the proof.

Lemma 7.2. Let $\Lambda=\mathcal{J}_{0}$, and let $\tilde{\Lambda}$ be a Julia arc from the finite family $\mathcal{F}$ guaranteed by Lemma 7.1. Then there exists a piecewise dynamical homeomorphism $\mathcal{T}$ of $\Lambda \backslash\{\alpha\}$ onto $\tilde{\Lambda} \backslash\left\{z_{1}, z_{2}\right\}$.

Proof. If, in the notation of Lemma 7.1, $\tilde{z}_{1}=\tilde{z}_{2}$, the present lemma is trivial. Otherwise, the intersection of $\tilde{\Lambda}$ with $\partial U_{0}$ is an arc with dyadic endpoints. In the Böttcher coordinate of $U_{0}$ the intersection $\Lambda \cap\left(\partial U_{0} \backslash\{\alpha\}\right)$ corresponds to the open arc $I_{0}=\mathbb{T} \backslash\{1\}$, and the intersection $\tilde{\Lambda} \cap\left(\partial U_{0} \backslash\left\{\tilde{z}_{1}, \tilde{z}_{2}\right\}\right)$ corresponds to an open arc $I_{1}$ on $\mathbb{T}$. Thus we can apply Lemma 5.1 to get a piecewise linear map $\tau$ from $I_{0}$ onto $I_{1}$ with only dyadic break points and with slopes that are integer powers of 2 . The map $\tau$ corresponds to a piecewise dynamical map $\mathcal{T}$ from $\Lambda$ onto $\tilde{\Lambda}$ as described in Section 5 , specifically in Lemma 5.4.

Let $\Lambda=\mathcal{J}_{0}$. For $n$ large as in Lemma 7.1, we consider a tiling of $\mathcal{J}$ into the Julia $\operatorname{arcs} J_{i}, i=1,2, \ldots, 2^{n+1}$, of $\Pi_{n}$. For each $i=$ $1,2, \ldots, 2^{n+1}$, there exists $n_{i} \in\{n, n+1\}$ such that $f^{n_{i}}$ maps $J_{i}$ onto $\Lambda$. Let $\tilde{J}_{i}=\xi\left(J_{i}\right)$, and $\tilde{\Lambda}_{i}=f^{M_{i}}\left(\tilde{J}_{i}\right)$, where $M_{i}$ is the constant from Lemma 7.1 corresponding to $\tilde{J}_{i}$. Now, let us replace the map $\xi$ on $J_{i} \backslash\left\{z_{1}^{i}, z_{2}^{i}\right\}$, where $z_{1}^{i}, z_{2}^{i}$ are the endpoints of $J_{i}$, by the map $\tau_{i}=$ $f^{-M_{i}} \circ \mathcal{T}_{i} \circ f^{n_{i}}$, where $\mathcal{T}_{i}$ is the piecewise dynamical map from $\Lambda \backslash\{\alpha\}$ onto int $\tilde{\Lambda}_{i}$ that comes from Lemma 7.2 . Pasting these $2^{n+1}$ maps together, we obtain a global piecewise dynamical map $\tau \in \operatorname{Dyn}(\mathcal{J})$.

We next prove that $\tau$ is quasisymmetric on $\mathcal{J}$ with a controlled distortion function $\eta$ by showing that it has a quasiconformal extension to the whole plane with a controlled dilatation $K$. We do this by showing that $\tau$ can be quasiconformally extended into each bounded Fatou component and into the basin at infinity, so that the dilatation is controlled. Let $U$ be an arbitrary bounded Fatou component of $f$. If 
the dynamical distance $m$ from $U$ to $U_{0}$ is greater than $\lfloor(n+1) / 2\rfloor$, the map $\tau$ has a conformal extension into $U$. Indeed, it is defined in $\partial U$ as a single element of the pseudo-group $\Upsilon_{f}$. Assume now that $m$ is at most $n$. Then the boundary of $U$ is partitioned by its intersection with the elements from $\Pi_{n}$ into the dyadic intervals of level $\lfloor(n+1) / 2\rfloor-m$. Let $t$ be the piecewise dynamical map of $\partial U$ that is the restriction of $\tau$ to $\partial U$. It agrees with $\xi$ at the endpoints of the dyadic intervals above. Now we need two more lemmas concerning circle maps.

For a homeomorphism $\xi$ of $\mathbb{T}$ and a finite set $E \subset \mathbb{T}$ we denote by $\xi_{E}$ the linear interpolation of the restriction $\left.\xi\right|_{E}$ of $\xi$ to $E$.

Lemma 7.3. For any distortion homeomorphism $\eta$, there exists a distortion homeomorphism $\eta^{\prime}$ with the following property. If $\xi$ is an $\eta$ quasisymmetric map of the unit circle $\mathbb{T}$ and $E$ is a finite subset of $\mathbb{T}$ such that all the complementary intervals of $E$ in $\mathbb{T}$ have the same angular length, then $\xi_{E}$ is $\eta^{\prime}$-quasisymmetric.

Proof. Let $d$ denote the angular distance on $\mathbb{T}$. Since $\xi$ is $\eta$-quasisymmetric, [He, Proposition 10.8] gives that there exists a constant $L \geq 1$ that depends only on $\eta$ such that for any three distinct points $o, p, q \in E$ with $d(o, p)=d(o, q)$, we have

$$
d(\xi(o), \xi(p)) \leq L d(\xi(o), \xi(q)) .
$$

Let $a$ denote the angular length of each complementary interval of $E$. To verify that $\xi_{E}$ is quasisymmetric with a distortion function that depends only on $\eta$, it is enough to check that there exists a constant $C \geq 1$ that depends only on $\eta$ and has the following property. If $o, p$, and $q$ are arbitrary points on $\mathbb{T}$ with $d(o, p)=d(o, q)=\delta$, for some $\delta>0$, then $d\left(\xi_{E}(o), \xi_{E}(p)\right) \leq C d\left(\xi_{E}(o), \xi_{E}(q)\right)$. We consider the following cases.

Case 1: $\delta \leq a$ and $o, p, q$ are contained in the same complementary interval of $E$. This case is trivial and $C=1$ because $\xi_{E}$ is linear on each such interval.

Case 2: $\delta \leq a$ and $o, p, q$ are not contained in the same complementary interval. We assume that $o$ and $p$ are contained in the closure of the same complementary interval $I$ of $E$ and $q$ is in the adjacent interval $I^{\prime}$. Let us assume that $\xi_{E}$ scales $I$ by $s$ and it scales $I^{\prime}$ by $s^{\prime}$. We know from (7.1) that $s / L \leq s^{\prime} \leq L s$. Let $c \in E$ be the common point of the intervals $I$ and $I^{\prime}$. Then

$d\left(\xi_{E}(o), \xi_{E}(q)\right)=d\left(\xi_{E}(o), \xi_{E}(c)\right)+d\left(\xi_{E}(c), \xi_{E}(q)\right)=s d(o, c)+s^{\prime} d(c, q)$.

For the last expression we have

$$
s d(o, q) / L \leq s d(o, c)+s^{\prime} d(c, q) \leq \operatorname{Lsd}(o, q) .
$$


Combining this with the assumptions that $d(o, p)=d(o, q)$ and that $\xi_{E}$ scales $I$ by $s$, we conclude that

$$
d\left(\xi_{E}(o), \xi_{E}(p)\right) / L \leq d\left(\xi_{E}(o), \xi_{E}(q)\right) \leq L d\left(\xi_{E}(o), \xi_{E}(p)\right)
$$

and so $C=L$ in this case.

Case 3: $a<\delta \leq 3 a$. This case reduces to a repeated application of the proof of Case 2 at most 3 times. We conclude that $C=L+L^{2}+L^{3}$ works in this case.

Case 4: $\delta>3 a$. Let $I_{o}, I_{p}$, and $I_{q}$ be the closures of the complementary intervals of $E$ that contain $o, p$, and $q$, respectively. Since $\xi_{E}$ agrees with $\xi$ at the endpoints of each such interval, there exist $o^{\prime}, p^{\prime}$, and $q^{\prime}$ in $I_{o}, I_{p}$, and $I_{q}$, respectively, such that $\xi_{E}(o)=\xi\left(o^{\prime}\right), \xi_{E}(p)=\xi\left(p^{\prime}\right)$, and $\xi_{E}(q)=\xi\left(q^{\prime}\right)$. Now,

$$
\begin{aligned}
\frac{d\left(\xi_{E}(o), \xi_{E}(p)\right)}{d\left(\xi_{E}(o), \xi_{E}(q)\right)} & =\frac{d\left(\xi\left(o^{\prime}\right), \xi\left(p^{\prime}\right)\right)}{d\left(\xi\left(o^{\prime}\right), \xi\left(q^{\prime}\right)\right)} \leq \eta\left(\frac{d\left(o^{\prime}, p^{\prime}\right)}{d\left(o^{\prime}, q^{\prime}\right)}\right) \\
& \leq \eta\left(\frac{\delta+2 a}{\delta-2 a}\right) \leq \eta(5),
\end{aligned}
$$

and the claim follows in this case with $C=\eta(5)$.

Lemma 7.4. Let $\mathcal{L}$ be a finite family of orientation preserving piecewise linear homeomorphisms between intervals of $\mathbb{T}$. Let $\xi$ be an orientation preserving homeomorphism of $\mathbb{T}$ and let $E$ be a finite subset of $\mathbb{T}$ such that all complementary intervals of $E$ in $\mathbb{T}$ have the same length. Suppose that $t$ is an orientation preserving piecewise linear homeomorphism of $\mathbb{T}$ that agrees with $\xi$ on the set $E$. Moreover, assume that for each complementary interval I of $E$ there are $M, n \in \mathbb{N}_{0}$ with $g^{M} \circ t \circ g^{-n}$ being an element of $\mathcal{L}$ defined on $g^{n}(I)$. Then there exists a constant $L \geq 1$ that depends only on $\mathcal{L}$ such that the map $\xi_{E}^{-1} \circ t$ is L-bi-Lipschitz.

Proof. The assumption implies, in particular, that there is a finite family $\mathcal{L}_{0}$ of orientation preserving linear maps between intervals of $\mathbb{T}$, that depends only on $\mathcal{L}$, such that for each complementary interval $I$ of $E$ in $\mathbb{T}$ the map $g^{M} \circ \xi_{E} \circ g^{-n}$ is an element of $\mathcal{L}_{0}$. Therefore the map $g^{n} \circ \xi_{E}^{-1} \circ t \circ g^{-n}$ is a homeomorphism of $g^{n}(I)$ that belongs to a finite family, depending only on $\mathcal{L}$, of orientation preserving piecewise linear homeomorphisms. Hence there exists $L \geq 1$ that depends only on $\mathcal{L}$ such that $g^{n} \circ \xi_{E}^{-1} \circ t \circ g^{-n}$ is an $L$-bi-Lipschitz homeomorphism of $g^{n}(I)$. Since $g$ is the scaling map (by the factor 2) in the angular metric, the map $\xi_{E}^{-1} \circ t$ is $L$-bi-Lipschitz on each $I$, and therefore on all of $\mathbb{T}$. 
Lemmas 7.3 and 7.4 imply that the restriction of the map $\tau$ to the boundary of each bounded Fatou component $U$ has a $K^{\prime}$-quasiconformal extension to $U$, where $K^{\prime}$ depends only on $\eta$. The set $E$ in Lemma 7.3 is the set of dyadic points at level $\lfloor(n+1) / 2\rfloor-m$, where $m$ is the dynamical distance from $U$ to $U_{0}$. The finite family $\mathcal{L}$ in Lemma 7.4 comes from Lemmas 7.1 and 7.2. Indeed, Lemma 7.1 guarantees the existence of a finite family $\mathcal{F}$ of subsets of $\mathcal{J}$ such that for any Julia arc $J \in \Pi_{n}$ we have $\tilde{\Lambda}=f^{M}(\xi(J)) \in \mathcal{F}$ for some $M \in \mathbb{N}_{0}$. Lemma 7.2 then gives a piecewise dynamical map $\mathcal{T}$ from $\Lambda \backslash\{\alpha\}$ onto $\tilde{\Lambda} \backslash\left\{z_{1}, z_{2}\right\}$, in the notations of that lemma. Because the family $\mathcal{F}$ is finite, the family of such maps $\mathcal{T}$ is finite. Now, if the defining external rays of $J$ land on the boundary of $U$ and if we assume for simplicity that $\Lambda=f^{n}(J)$ rather than $\Lambda=f^{n+1}(J)$, then we have $\mathcal{T}=f^{M} \circ \tau \circ f^{-n}$, where the inverse branches of $f$ are chosen appropriately. Passing to the Böttcher coordinates of $U$ and $\tilde{U}=\xi(U)$ we conclude that the map $g^{M-\tilde{m}} \circ t \circ g^{-(n-m)}$, belongs to a finite family $\mathcal{L}$, where $t$ is the conjugate map of the map $\tau$ by $\phi_{U}$, and $\tilde{m}$ is the dynamical distance from $\tilde{U}$ to $U_{0}$. Since $\xi_{E}$ is $\eta^{\prime}$-quasisymmetric and $\xi_{E}^{-1} \circ t$ is $L$-bi-Lipschitz, we conclude that $t=\xi_{E}\left(\xi_{E}^{-1} \circ t\right)$ is $\eta^{\prime} \circ L^{2}$-quasisymmetric, where $L^{2}$ is the scaling map by $L^{2}$. Thus, the Ahlfors-Beurling extension [BA] gives that there exists $K^{\prime} \geq 1$ that depends only on $\eta^{\prime}$ and $L$, and hence only on $\eta$, such that the map $t$ has a $K^{\prime}$-quasiconformal extension into $\mathbb{D}$. Conjugating back via Böttcher coordinates we conclude that $\tau$ has a $K^{\prime}$-quasiconformal extension into every bounded Fatou component $U$.

To deal with the unbounded component $U_{\infty}$, we first apply Lemma 3.2 and then proceed in the same way as for bounded Fatou components, i.e., using Lemmas 7.3 and 7.4. The finite set $E$ in this case is the set $\psi\left(D_{n}\right)$, where $\psi$ is the bi-Lipschitz map guaranteed by Lemma 3.2 . The crucial difference is that, unlike the case of bounded Fatou components, the boundary of $U_{\infty}$ touches itself and one has to be careful to preserve the basilica lamination. By Lemma 3.4, any topologically extendable homeomorphism $\xi$ of $\mathcal{J}$ induces a homeomorphism $\xi_{\infty}$ of $\mathbb{T}$ that preserves the basilica lamination. By Lemma 4.1, $\xi_{\infty}$ is $\eta^{\prime}-$ quasisymmetric (with $\eta^{\prime}$ depending only on $\eta$ ). As $\xi$ is replaced by a piecewise dynamical map $\tau$, the map $\xi_{\infty}$ is replaced by a Thompsonlike element $\tau_{\infty}$ satisfying $\psi_{\infty} \circ \tau_{\infty}=\tau \circ \psi_{\infty}$. Such a map $\tau_{\infty}$ necessarily respects the basilica lamination. By Lemmas 7.3 and 7.4 , the map $\tau_{\infty}$ is $\eta^{\prime \prime}$-quasisymmetric with $\eta^{\prime \prime}$ that depends only on $\eta^{\prime}$ and hence only on $\eta$. Therefore it extends to a $K^{\prime \prime}$-quasiconformal homeomorphism of $\mathbb{C} \backslash \mathbb{D}$ (with $K^{\prime \prime}$ depending only on $\eta^{\prime \prime}$, and ultimately, only on $\eta$ ). 
Thus, the map $\tau$ has a $K^{\prime \prime}$-quasiconformal extension to $U_{\infty}$ as well, where $K^{\prime \prime}$ depends only on $\eta$. Putting this together, we obtain a $K$ quasiconformal extension of $\tau$ from $\mathcal{J}$ into each Fatou component of $f$, where $K=\max \left\{K^{\prime}, K^{\prime \prime}\right\}$. We denote this extension by $\tau$ as well. As above, the polynomial $f$ is postcritically finite, and, according to [Jo], the Julia set $\mathcal{J}$ is removable for quasiconformal maps. The map $\tau$ is hence $K$-quasiconformal in the whole complex plane, and therefore $\eta^{\prime}$ quasisymmetric for some $\eta^{\prime}$ that depends only on $\eta$. Its restriction $\tau$ to $\mathcal{J}$ is thus also $\eta^{\prime}$-quasisymmetric.

Lemma 7.5. Any piecewise dynamical map $\tau$ of $\mathcal{J}$ belongs to the group $\hat{T}$ generated by $T$ and $\iota$.

Proof. It is proved in Lemma 6.2 that the group $\hat{T}$ acts transitively on the set of bounded Fatou components. Suppose that $\tau\left(\partial U_{0}\right)=\partial \tilde{U}_{0}$ and let $g \in \hat{T}$ be such that $g\left(\partial U_{0}\right)=\partial \tilde{U}_{0}$. Then $g^{-1} \circ \tau$ keeps $\partial U_{0}$ invariant, and so, without loss of generality, we assume that $\tau$ keeps $\partial U_{0}$ invariant. Note that Lemma 2.1 implies that such $\tau$ sends the root of each $U \neq U_{0}$ to the root of $\tilde{U}=\tau(U)$.

Let $U$ be any bounded Fatou component of $f$ and $\tilde{U}=\tau(U)$. Let $\phi_{U}$ and $\phi_{\tilde{U}}$ be the Böttcher coordinates of $U$ and $\tilde{U}$, respectively. Since $\tau$ is assumed to be piecewise dynamical, the restriction of the map $\phi_{\tilde{U}} \circ \tau \circ \psi_{U}$ to the unit circle is an element of the Thompson group $T$ of the unit circle. Here $\psi_{U}=\phi_{U}^{-1}$. Moreover, this map respects the Böttcher coordinate for all but finitely many bounded Fatou components $U$. This follows immediately from the fact that there are only finitely many global cut-points used in the definition of a piecewise dynamical map. Let $t$ be the smallest subtree of $\mathfrak{T}$ that contains $U_{0}$ and such that $\tau$ respects the Böttcher coordinate of every bounded Fatou component $U$ that is not a vertex of $t$. Let $k$ be the number of vertices of $t$. We prove by induction on $k$ that there is an element $g$ of $\hat{T}$ such that $\tau=g \in \hat{T}$. If $k=1$, then there exists $\theta \in T$ such that $\theta^{-1} \circ \tau$ is the identity element on $\partial U_{0}$. Moreover, since $\theta$ respects the Böttcher coordinate of each bounded Fatou component $U \neq U_{0}$, then $\theta^{-1} \circ \tau$ is necessarily the identity in the Böttcher coordinate of every $U$, and so it is the identity.

Now suppose the result is true for $k-1$. Let $U$ be a leaf of $t$, i.e., a degree one vertex, and let $\tilde{U}$ be the leaf of $\tilde{t}$ that corresponds to $U$ under $\tau$. Let $p$ be the unique path from $U_{0}$ to $U$ in $t$. By Lemma 6.2, there is an element $g_{U} \in \hat{T}$ such that $g_{U}(\partial U)=\partial U_{0}$ and $g_{U}$ respects the Böttcher coordinates of all bounded Fatou components $V$ with the possible exception of $V$ being a vertex of $p$. Likewise, there is an element $g_{\tilde{U}} \in \hat{T}$ that has the same properties with respect to $\tilde{U}$ 
and $\tilde{t}$. Also, $g_{U}$ and $g_{\tilde{U}}$ respect the Böttcher coordinates of $U$ and $\tilde{U}$, respectively.

Then the restriction of $g_{\tilde{U}} \circ \tau \circ g_{U}^{-1}$ to $\partial U_{0}$ equals to the restriction of an element $\theta_{U} \in T$, and hence $g_{\tilde{U}} \circ \tau \circ g_{U}^{-1} \circ \theta_{U}^{-1}$ is the identity on $\partial U_{0}$. Moreover, since $\tau$ takes the root of $U$ to the root of $\tilde{U}$, the map $\theta_{U}$ fixes the root $\alpha$ of $U_{0}$. The map $g=g_{U}^{-1} \circ \theta_{U} \circ g_{U}$ is then an element of $\hat{T}$ that keeps $U$ invariant, fixes the root of $U$, and respects the Böttcher coordinate of every $V \neq U$. In the Böttcher coordinates of $U$ and $\tilde{U}$, the map $\tau \circ g^{-1}$ is the identity. Also, it is the identity in Böttcher coordinates of every vertex $V$ that is not in $t$. Now we can apply the induction.

Recall that in $\mathrm{BF}$ the authors studied the Thompson-like group $T_{\alpha}^{b}$ of piecewise linear homeomorphisms of the unit circle that preserve the basilica lamination. Obviously, our group $\hat{T}$ generated by $T$ and $\iota$ is isomorphic to a subgroup of $T_{\boldsymbol{\alpha}}^{b}$. The following is an immediate corollary of Lemmas 5.6 and 7.5 .

Corollary 7.6. The groups $\hat{T}$ and $T_{\boldsymbol{\alpha}}^{b}$ are isomorphic.

To finish the proof of Theorem 1.1 we need to show that $\tau$ approaches $\xi$ on $\mathcal{J}$ uniformly as $n$ goes to infinity. Indeed, as one moves further away from the vertex of $\mathfrak{T}$ that corresponds to $U_{0}$, the diameters of the corresponding bounded Fatou components go to 0. This follows from the fact that $f$ is hyperbolic. Moreover, the diameter of each $J_{k} \in \Pi_{n}$ goes to 0 as $n \rightarrow \infty$. This, along with the fact that $\tau$ agrees with $\xi$ at all the preimages of $\alpha$ under $f^{n}$, finishes the proof of Theorem 1.1 .

\section{REFERENCES}

[AIM] K. Astala, T. Iwaniec, G.M. Martin, Elliptic partial differential equations and quasiconformal mappings in the plane, Princeton Univ. Press, Princeton, NJ, 2009.

[BF] J. Belk, B. Forrest, A Thompson group for the basilica, Groups Geom. Dyn. 9 (2015), no. 4, 975-1000.

[BA] A. Beurling, L. Ahlfors, The boundary correspondence under quasiconformal mappings, Acta Math. 96 (1956), 125-142.

[BKM] M. Bonk, B. Kleiner, S. Merenkov, Rigidity of Schottky sets, Amer. J. Math. 131 (2009), no. 2, 409-443.

[BLM] M. Bonk, M. Lyubich, S. Merenkov, Quasisymmetries of Sierpiński carpet Julia sets, Adv. Math. 301 (2016), 383-422.

[BM] M. Bonk, S. Merenkov, Quasisymmetric rigidity of square Sierpinski carpets, Ann. of Math. (2) 177 (2013), no. 2, 591-643.

[CC] A. Candel, L. Conlon, Foliations I. Graduate Studies in Mathematics, 23. American Mathematical Society, Providence, RI, 2000. xiv+402 pp. 
[CFP] J. W. Cannon, W. P. Floyd, W. R. Parry, Introductory notes on Richard Thompson's groups, Enseign. Math. (2) 42 (1996), no. 3-4, 215-256.

[CG] L. Carleson, Th.W. Gamelin, Complex dynamics, Springer, New York, 1993.

[D] A. Douady, Description of compact sets in $\mathbb{C}$. In: "Topological Methods in Modern Mathematics, A Symposium in Honor of John Milnor's 60th Birthday", Publish or Perish, 1993.

[DH] A. Douady, J. H. Hubbard, Étude dynamique des polynômes complexes. Publication Mathematiques d'Orsay, 84-02 and 85-04.

[He] J. Heinonen, Lectures on analysis on metric spaces, Springer-Verlag, New York, 2001.

[Jo] P. Jones, On Removable sets for Sobolev spaces in the plane, IMS Preprint Server, http://www.math.stonybrook.edu/preprints/ims91-22.pdf.

[L] M. Lyubich, Conformal geometry and dynamics of quadratic polynomials. www.math.stonybrook/ mlyubich/book.pdf.

$[\mathrm{Me}]$ S. Merenkov, A Sierpinski carpet with the co-Hopfian property, Invent. Math., Vol. 180, Issue 2 (2010), 361-388.

[Mi] J. Milnor, Geometry and dynamics of quadratic rational maps, Experiment. Math. 2 (1993), 37-83.

[Th] W. Thurston, On the geometry and dynamics of iterated rational maps. In: Complex Dynamics: Friends and Families, pp. 3-110. Editors: D. Schleicher, A. K. Peters.

[Vä] J. Väisälä, Lectures on n-dimensional quasiconformal mappings, Lecture Notes in Mathematic 229, Springer, Berlin-Heidelberg-New York, 1971.

Mikhail Lyubich, Institute for Mathematical Sciences, Stony Brook University, STONY Brook, NY 11794, USA

E-mail address: mlyubich@math.stonybrook.edu

Sergei Merenkov, Department of Mathematics, City College of New York and CUNY Graduate Center, New York, NY 10031, USA

E-mail address: smerenkov@ccny.cuny.edu 